\title{
Expériences de laboratoire sur le comportement thermo-hydro-mécanique de matériaux argileux remaniés gonflants et non gonflants
}

Euro-Géomat Consulting, 51, route d'Olivet 45100 Orléans

F. PLAS

Andra

Service matériaux Direction scientifique Parc de la Croix-Blanche, 1/7, rue Jean-Monnet, 92298 Châtenay-Malabry
Des essais ont permis d'analyser aux niveaux macroscopique et microscopique, le comportement thermo-hydro-mécanique des argiles gonflantes et non gonflantes. Le comportement mécanique a été étudié au moyen d'essais cedométriques cycliques avec et sans mesure des contraintes radiales, ainsi que d'essais de compression triaxiale non drainés à différentes pressions de confinement. L'influence de la pression de consolidation sur les textures a été analysée sur la base de résultats d'essais de porosimétrie au mercure et de BET. Ces essais ont permis de mettre en évidence un comportement élasto-plastique en charge pour les différentes roches argileuses gonflantes et non gonflantes. Dans le cas des roches gonflantes, la décharge et la recharge s'accompagnent de boucles d'hystérésis traduisant l'existence de déformations irréversibles et un comportement élasto-plastique. Le coefficient $\mathrm{k}_{\text {。 }}$ pour les différentes roches peut être évalué avec une relation linéaire en fonction du rapport de contraintes $\mathrm{q} / \mathrm{p}^{\prime}$. Ainsi, pour les états de contrainte normalement consolidés, les variations des contraintes extérieures sont équilibrées par l'évolution des contraintes de contact : $\delta \sigma_{\text {ext }}=\delta \sigma_{\mathrm{c}^{*}}$. En revanche, pour les états de contrainte surconsolidés et dans le cas des roches gonflantes, les variations des contraintes extérieures sont reprises par les contraintes physicochimiques de répulsion $\delta \sigma_{\mathrm{eq}}=\delta \sigma_{\mathrm{n}-\mathrm{A}}$. Pour l'étude du comportement thermo-mécanique, nous avons réalisé d'une part, des essais isothermes sur des chemins œedométriques et triaxiaux non drainés et, d'autre part, des cycles thermiques sous chargement mécanique constant à l'état normalement consolidé et à l'état surconsolidé sur des chemins œdométriques.

L'anisotropie des déformations sous sollicitations thermiques et les déformations irréversibles des textures des roches argileuses ont été étudiées. Elles permettent ainsi d'associer à ces sollicitations thermo-mécaniques un opérateur tensoriel : la contrainte thermique $\sigma_{\text {. }}$. Les principaux phénomènes liés au comportement hydromécanique des roches argileuses fortement compactées ont été mis en évidence par des essais œdométriques et triaxiaux à succion constante et par des cycles de sorption-désorption. Ainsi les essais de sorptiondésorption mettent en évidence deux domaines : un domaine quasi saturé dans lequel les déformations sont isotropes et un domaine non saturé caractérisé par des déformations réversibles anisotropes. Un opérateur tensoriel de contrainte capillaire $\sigma_{\text {cap }}$ est proposée pour traduire ces déformations. Ainsi, dans le cas général de sollicitations thermo-hydro-mécaniques, les variations de contraintes extérieures sont équilibrées par des contraintes de contact pour les états de contrainte normalement consolidés (OCR $=1$ ) ou de répulsion pour les états de contrainte surconsolidés (OCR $>1$ ), par des 
contraintes thermiques et par des contraintes capillaires : $\underline{\sigma_{\text {ext }}}=\underline{\sigma_{c}}+\sigma_{\text {cap }}+\underline{\sigma_{T}}(\mathrm{OCR}=1)$ et

$\underline{\sigma_{\text {ext }}}=\underline{\sigma_{\mathrm{R}}}+\sigma_{\text {cap }}+\underline{\sigma_{\mathrm{T}}}(\mathrm{OCR}>1)$

\section{Laboratory experiments on the thermo-hydro-mechanical behaviours of swelling and unswelling clayey rocks}

Some tests allowed to analyse at macroscopic and microscopic levels, the thermo-hydro-mechanical behaviour of swelling and unswelling clayey rocks. The mechanical behaviour was studied by oedometric cyclic tests with and without measurement of the radial stress, as well as undrained compression triaxial tests at different confining pressures. The influence of the consolidation pressure on the textures was analysed from results of porosimeter and BET tests. These tests allowed to point out an elasto-plastic behaviour during the loading for different swelling and unswelling clayey rocks. In the case of the swelling rocks, the unloading and the reloading curves showed hysteresis loops, which proved the existence of irreversible strains and an elasto-plastic behaviour. The $\mathrm{k}_{\mathrm{o}}$ coefficient for the different rocks could be valued by linear relation with the stresses ratio $\mathrm{q} / \mathrm{p}$ '. Thus for the normally consolidated states, the variations of the external stress are level-headed by the evolution of the stress of contact : $\delta \sigma_{\text {en }}=\delta \sigma_{\text {. }}$. In return for the overconsolidated states and in the case of the swelling clayey rocks, the variations of the external stress are compensated by the physico-chemical repulsion stress : $\delta \sigma_{\text {en }}=\delta \sigma_{1}$. . To study the thermo-mechanical behaviour, we performed on one hand isothermal tests on some oedometric and undrained triaxial paths, and on the other hand thermal cycles under mechanical constant loading at normally and over consolidated states on some oedornetric paths. The anisotropy of the strain under thermal solicitations and the irreversible strain of clayey rock textures were studied. Thus they allow to associate a tensorial operator, the thermal stress $\sigma$. to thermo-mechanical solicitations. The main phenomena due to the hydro-mechanical behaviour of highly compacted clayey rocks were pointed out by oedometric and trixial tests at constant suction and by sorption desorption cycles. So sorption desorption tests show two domains : a quasi saturated domain for which the strains are isotropic and a no saturated one characterised by reversible anisotropic strain. A tensor of capillary stress $\sigma_{\text {en }}$ is suggested in order to translate these strains. So in the general case of thermo-hydro-mechanical solicitations, the variations of external stress are equilibrated by the contact stress $(O C R=1)$ or repulsion stress (OCR $>1$ ), and by the thermal stress and the capillary stress: $\underline{\sigma_{\text {ext }}}=\underline{\sigma_{c}}+\sigma_{\text {cap }}+\underline{\sigma_{1}}(O C R=1)$ and $\underline{\sigma_{\text {ext }}}=\underline{\sigma_{\mathrm{R}}}+\sigma_{\mathrm{cap}}+\underline{\sigma_{\mathrm{T}}}(\mathrm{OCR}>1)$ 


\section{NOTATIONS}

\begin{tabular}{|c|c|}
\hline & : indice des vides. \\
\hline OCR & : degré de consolidation. \\
\hline$\sigma_{e x t}$ & : contrainte totale extérieure $(\mathrm{Pa})$. \\
\hline$\sigma_{c}$ & : contrainte de contact $(\mathrm{Pa})$. \\
\hline$\sigma_{\mathrm{f}}$ & $\begin{array}{l}\text { contrainte physico-chimique de } \\
\text { répulsion }(\mathrm{Pa}) \text {. }\end{array}$ \\
\hline$\sigma_{\text {cap }}$ & : contrainte capillaire (Pa) \\
\hline$\sigma_{1}$ & : contrainte thermique $(\mathrm{Pa})$. \\
\hline$\sigma_{1}$ & : contrainte verticale $(\mathrm{Pa})$. \\
\hline$\sigma_{2}$ & : contrainte radiale $(\mathrm{Pa})$. \\
\hline$q=\sigma_{1}^{\prime}-\sigma_{2}^{\prime}$ & : déviateur de contrainte $(\mathrm{Pa})$. \\
\hline$p^{\prime}=\left[\sigma_{1}^{\prime}+2 \cdot \sigma_{2}^{\prime}\right] / 3$ & : pression moyenne effective ( \\
\hline $\mathrm{k}_{\mathrm{o}}=\sigma_{2}^{\prime} / \sigma_{1}^{\prime}$ & $\begin{array}{l}\text { : coefficient de poussée des terres } \\
\text { au repos. }\end{array}$ \\
\hline$M=q / p^{\prime}$ & : pente d'état critique. \\
\hline$E$ & : module d'Young ( $\mathrm{Pa}$ ), \\
\hline$v$ & : coefficient de Poisson. \\
\hline s & : succion $(\mathrm{Pa})$. \\
\hline $\mathrm{h}_{\mathrm{r}}$ & : humidité relative. \\
\hline M & : masse molaire (g.mol-1). \\
\hline $\mathrm{T}$ & : température $\left({ }^{\circ} \mathrm{K}\right)$. \\
\hline$\rho_{w}$ & $\begin{array}{l}\text { : masse volumique de l'e } \\
\left(10^{3} \mathrm{~kg} / \mathrm{m}^{3}\right) \text {. }\end{array}$ \\
\hline & pression de consolidation \\
\hline
\end{tabular}

gonflante de Couy et gonflante de Boom, ceux de Baldi et al. (1987) sur l'argile de Boom, ceux de Borgesson et al. (1993) sur une bentonite MX 80 et ceux de Lingnau et al. (1993) sur des mélanges sable-smectite. Dans le cas des roches partiellement saturées les travaux sont peu nombreux, le comportement hydro-mécanique a essentiellement été étudié à partir d'essais de compression simple sur des échantillons préparés dans des enceintes à humidité relative contrôlée, Colback et al. (1965) et Ballivy et al. (1992). Les premières études portant sur le couplage thermo-mécanique des roches saturées ont été réalisées par Baldi et al. (1990). Del Olmo et al. (1996) ont étudié le comportement thermo-mécanique de trois formations argileuses profondes : Boom en Belgique, Pasquasia en Italie et SS-1 IC-1 en Espagne.

Cet article a pour objectif de faire un inventaire des principaux mécanismes liés aux sollicitations mécanique, thermo-mécanique et hydro-mécanique sur des roches argileuses remaniées. La démarche consiste à s'appuyer sur les résultats d'essais œdométriques cycliques, et d'essais triaxiaux à différentes températures pour analyser l'effet de la pression de consolidation, de cycles de température et de cycles de drainagehumidification sous un état de chargement constant.

\section{2}

\section{Comportement mécanique des roches argileuses remaniées, saturées}

\section{1}

\section{Comportement sur chemin œdométrique}

L'étude expérimentale a été réalisée avec un œdomètre à haute pression sans mesure de la contrainte radiale (Fig. 1) ou avec mesure de la contrainte radiale (Fig. 2). La première partie de l'étude a été menée sur quatre matériaux argileux de nature différente, à savoir: une kaolinite, une smectite, une argile du Bassin parisien et l'argile de Boom. Les caractéristiques de ces matériaux sont données dans le tableau I.

Les essais ont consisté à effectuer des cycles de "décharge-recharge » afin d'analyser le gonflement de chaque matériau. Les échantillons sont consolidés à une pression de $5 \mathrm{MPa}$, puis déchargés à une pression relativement faible $(0,15-0,45 \mathrm{MPa})$, ensuite rechargés à une pression de $10 \mathrm{MPa}$ et enfin complètement déchargés. Une contre-pression d'eau de $1 \mathrm{MPa}$ a été appliquée à l'échantillon durant l'essai afin d'éviter sa désaturation. Les résultats de ces essais présentés sur la figure 3 montrent les phénomènes suivants :

- les courbes du premier chargement sont des droites en coordonnées semi-logarithmique qui diffèrent suivant les matériaux, et le comportement est élasto-plastique ;

- les pentes de décharge sont plus fortes pour l'argile de Boom et la smectite ;

- les boucles d'hystérésis après la décharge à $5 \mathrm{MPa}$ sont plus aplaties pour la kaolinite et l'argile du Bassin parisien. Elles sont plus larges pour la smectite et l'argile de Boom.

Nous avons constaté que les différences de comportement entre les matériaux sont essentiellement al. (1993) sur l'argile de Boom et la smectite MX 80, les essais triaxiaux de Rousset (1987) sur les argiles non 


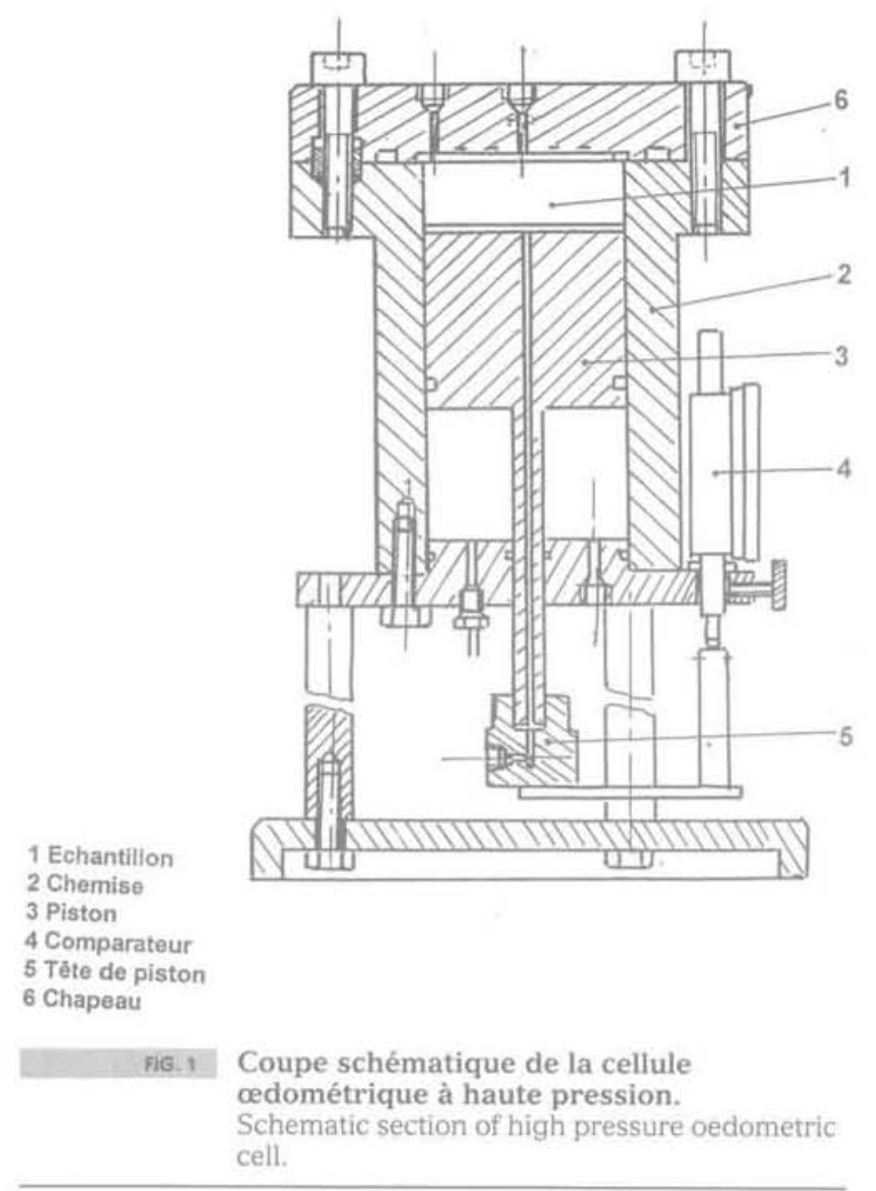

observées lors de la décharge. La smectite et, à un degré moindre, l'argile de Boom possèdent un gonflement beaucoup plus important que la kaolinite et I'argile du Bassin parisien. En effet, la diminution de la contrainte mécanique va provoquer le gonflement du matériau sous l'effet des forces non-équilibrées de
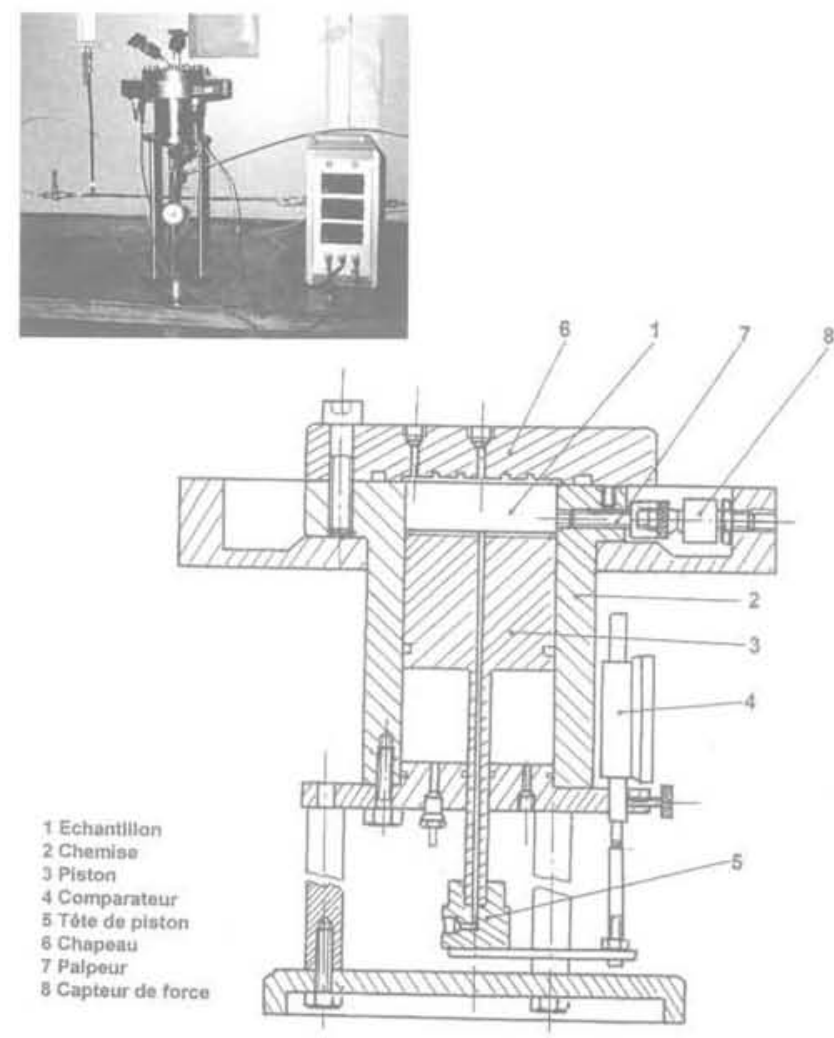

FiG. 2 Coupe schématique de la cellule œdométrique à haute pression avec mesure de la contrainte radiale. Schematic section of high pressure oedometric cell with radial stress measurement.

répulsion, Robinet et al. (1994). Ce gonflement augmente avec la pression de consolidation.

Les mesures des contraintes verticales et horizontales sur des chemins cycliques ont été réalisées sur une kaolinite et une smectite. Les résultats de ces essais sont traduits dans trois référentiels : e-Log $\sigma_{1}, \mathrm{q}-\mathrm{p}^{\prime}$ et

ThBisul Caractéristiques physiques et minéralogiques de l'argile du Bassin parisien, de la kaolinite, de la smectite et de l'argile de Boom.

Physical and mineralogical characteristics of Bassin parisien clay, kaolinite, smectite and Boom clay.

\begin{tabular}{|c|c|c|c|c|}
\hline Caractéristiques physiques & Argile du Bassin parisien & Kaolinite & Smectite & Argile de Boom \\
\hline $\begin{array}{l}\text { Densité des grains } \gamma_{s}\left(\mathrm{~g} / \mathrm{cm}^{3}\right) \\
\text { Surface spécifique }\left(B . E . T .\left(\mathrm{m}^{2} / \mathrm{g}\right)\right) \\
\text { Capacité d'échange de base (méq) } \\
\text { Limite de liquidité } W_{L}(\%) \\
\text { Limite de plasticité } W_{p}(\%) \\
\text { Indice de plasticité } I P=W_{L}-W_{p} \\
\text { Type d'argile }\end{array}$ & $\begin{array}{c}2,68 \\
33,5 \\
46 \\
52,5 \\
22,5 \\
30 \\
\text { non gonflante }\end{array}$ & $\begin{array}{c}2,5 \\
10,3 \\
- \\
40 \\
20 \\
20 \\
\text { non gonflante }\end{array}$ & $\begin{array}{l}2,75 \\
426 \\
64 \\
112 \\
50 \\
62 \\
\text { gonflante }\end{array}$ & $\begin{array}{l}2,67 \\
53-177 \\
20-40 \\
59-76 \\
22-26 \\
27-50 \\
\text { gonflante }\end{array}$ \\
\hline \multicolumn{5}{|l|}{ Composition minéralogique } \\
\hline $\begin{array}{l}\text { Kaolinite } \\
\text { Géothite } \\
\text { Calcite } \\
\text { Quartz } \\
\text { Ilite } \\
\text { Smectite } \\
\text { Moscovite } \\
\text { Feldspath et mica }\end{array}$ & $\begin{array}{l}- \\
- \\
- \\
- \\
-\end{array}$ & $\begin{array}{c}86 \% \pm 3 \\
- \\
- \\
6 \% \pm 1 \\
- \\
- \\
- \\
8 \% \pm 1\end{array}$ & $\begin{array}{c}4 \% \pm 2 \\
6 \% \pm 1 \\
2 \% \pm 1 \\
6 \% \pm 1 \\
- \\
80 \% \pm 1 \\
-\end{array}$ & $\begin{array}{l}30 \% \\
- \\
- \\
28 \% \\
20 \% \\
22 \% \\
- \\
-\end{array}$ \\
\hline
\end{tabular}




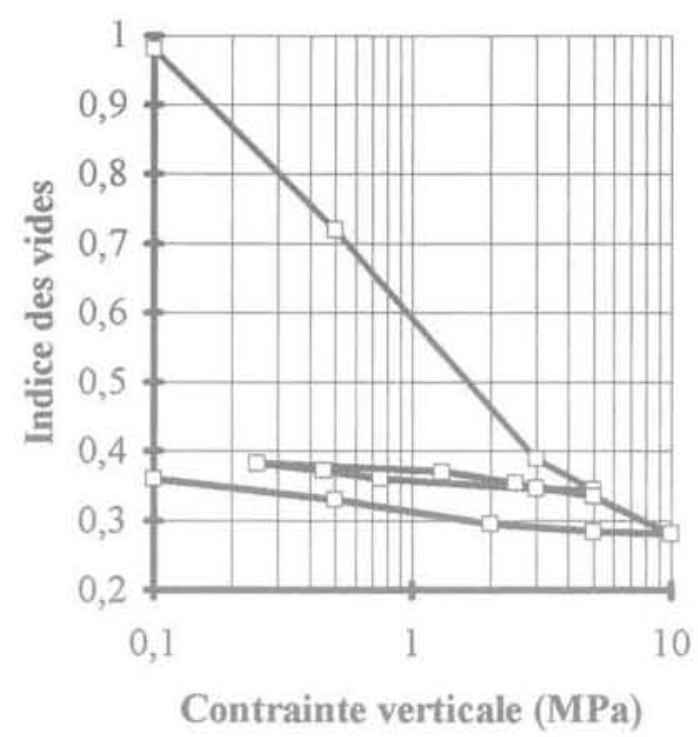

(a)

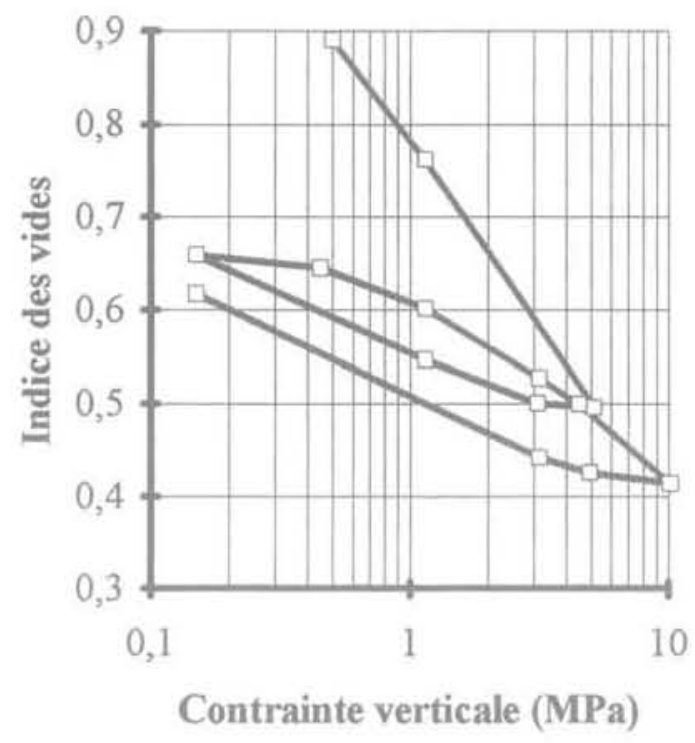

(c)

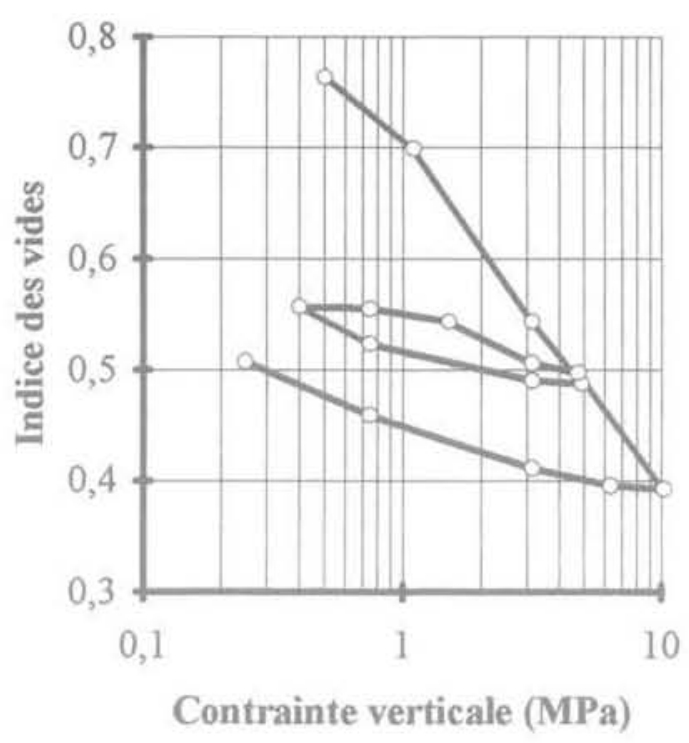

(b)

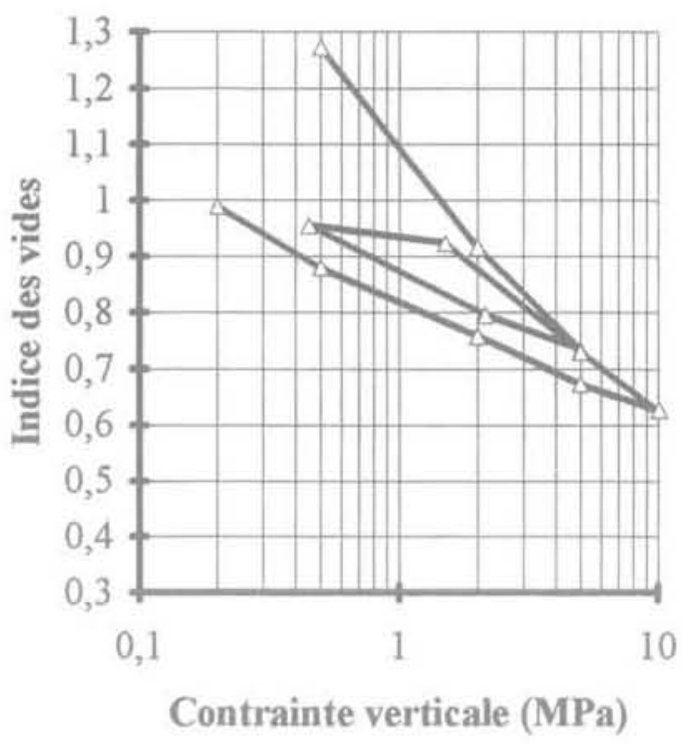

(d)

HG.3 Essais œdométriques cycliques sur des argiles remaniées saturées $\propto$ comparaison du comportement entre argiles gonflantes et non gonflantes ») (a) argile du Bassin parisien (b) kaolinite (c) argile de Boom (d) smectite.

Cyclic oedometer tests on remoulded saturated clays $\approx$ comparison of behaviour between swelling and unswelling clays » (a) Bassin parisien clay (b) kaolinite (c) Boom clay (d) smectite.

$k_{-}-q / p$ avec $q=\sigma_{1}^{\prime}-\sigma_{2}^{\prime}, p^{\prime}=\left[\sigma_{1}^{\prime}+2 \cdot \sigma_{2}^{\prime}\right] / 3$ et $k_{0}=\sigma_{2}^{\prime} / \sigma_{1}^{\prime}$ (Fig. 4 et 5). Ces résultats mettent en évidence:

- sur la kaolinite :

- un comportement réversible dans le plan e-Log $\sigma_{1}$ pour les états de contrainte surconsolidés,

- une boucle d'hystérésis très marquée dans le plan q-p' sur des cycles de décharge recharge, jusqu'à atteindre l'état normalement consolidé pour lequel le matériau retrouve le comportement du matériau normalement consolidé,

- une variation de $k_{o}$ réversible avec $q / p^{\prime}$;
- sur la smectite:

- un important gonflement après une décharge et une boucle d'hystérésis dans le plan e-Log $\sigma_{1}$,

- un comportement réversible dans le plan $q-p^{\prime}$ pour tous les états de contraintes normalement consolidés et surconsolidés.

Les variations expérimentales de $\mathrm{k}_{\mathrm{o}}$ avec le rapport $q / p^{\prime}$ pour la kaolinite et la smectite montrent une totale réversibilité de $k_{0}$ avec $\mathrm{q} / \mathrm{p}^{\prime}$ sur les chemins cycliques et des différences négligeables entre les deux matériaux. 

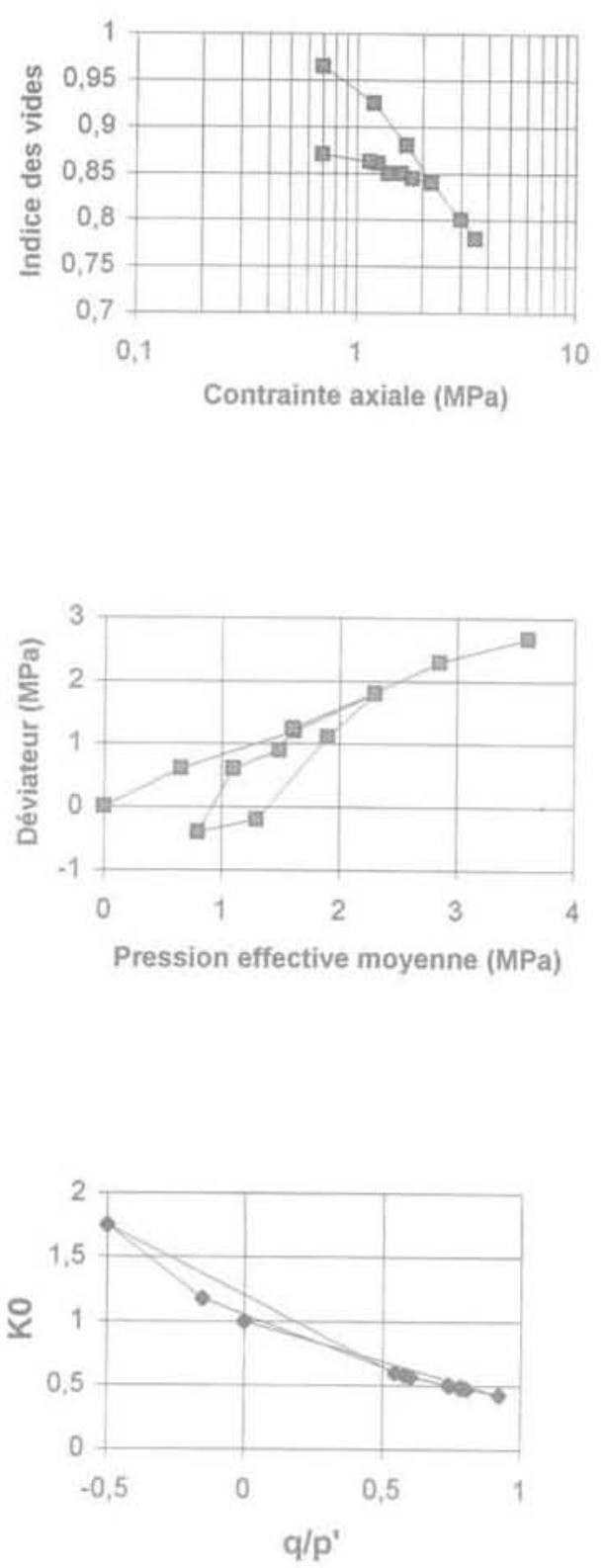

FG.4 Chemin cyclique œdométrique sur une kaolinite saturée. Cyclic oedometric path on saturated kaolinite.

La distribution porale totale des argiles étudiées est déterminée en s'appuyant sur deux méthodes dont le domaine d'application est fonction de la taille des pores. La frontière entre le passage d'une méthode à une autre est déterminée par le choix du porosimètre mercure qui dans notre cas ne permet pas d'atteindre des pores de diamètre inférieur à $100 \AA$. Ainsi pour les pores supérieurs à $100 \AA$, est utilisée la méthode de porosimétrie mercure associée à la technique de lyophilisation ou cryo-dessiccation. La lyophilisation des échantillons empêche toute fermeture des pores due à l'évacuation de l'eau lors du séchage à $105^{\circ} \mathrm{C}$ qui précède l'étude par porosimétrie mercure. Ainsi, la texture est parfaitement conservée. Toutefois, nos échantillons ayant une faible teneur en eau, le temps de lyophylisation n'a pas dépassé les soixante-douze heures. Quant aux pores dont la taille est inférieure à $100 \AA$, ils ont été étudiés à partir des isothermes de désorption d'azote.
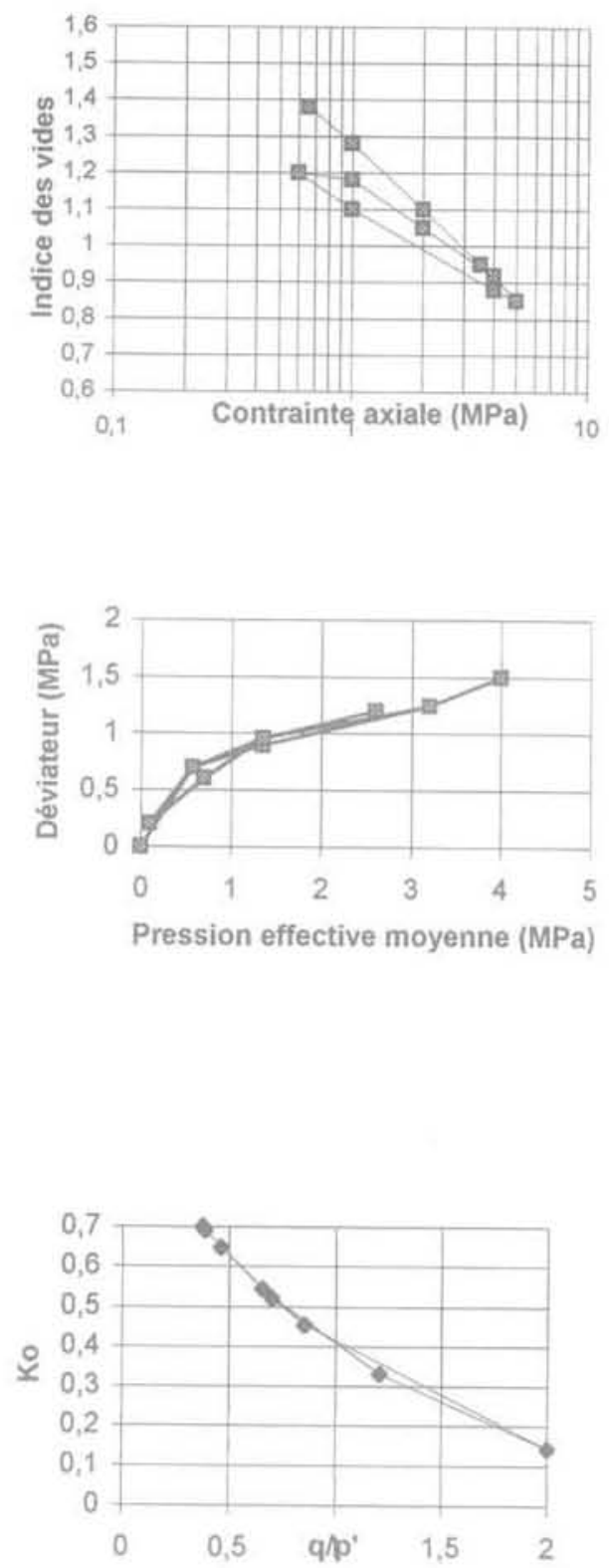

FG.5 Chemin cyclique cedométrique sur une smectite saturée.

Cyclic oedometric path on saturated smectite

Les distributions porales présentées sur les figures 6 et 7 correspondent à des échantillons compactés à une pression donnée, puis déchargés mécaniquement sur un chemin de sollicitation cedométrique. Les résultats obtenus ne reflètent donc pas exactement l'organisation porale de l'échantillon telle qu'elle devrait se présenter sous contrainte, mais ils nous permettent tout de même d'apprécier l'influence de la pression de consolidation sur l'organisation porale et surtout d'étudier les différents comportements en décharge, suivant la nature des argiles.

L'analyse des résultats d'essais de porosimétrie nous conduit à faire les observations suivantes :

- pour les deux argiles, la pression de consolidation semble ne pas influencer la microporosité (diamètre des pores inférieur à $100 \AA$ ). En effet, nous remarquons que les courbes de distribution porale (Fig. 6) sont qua- 

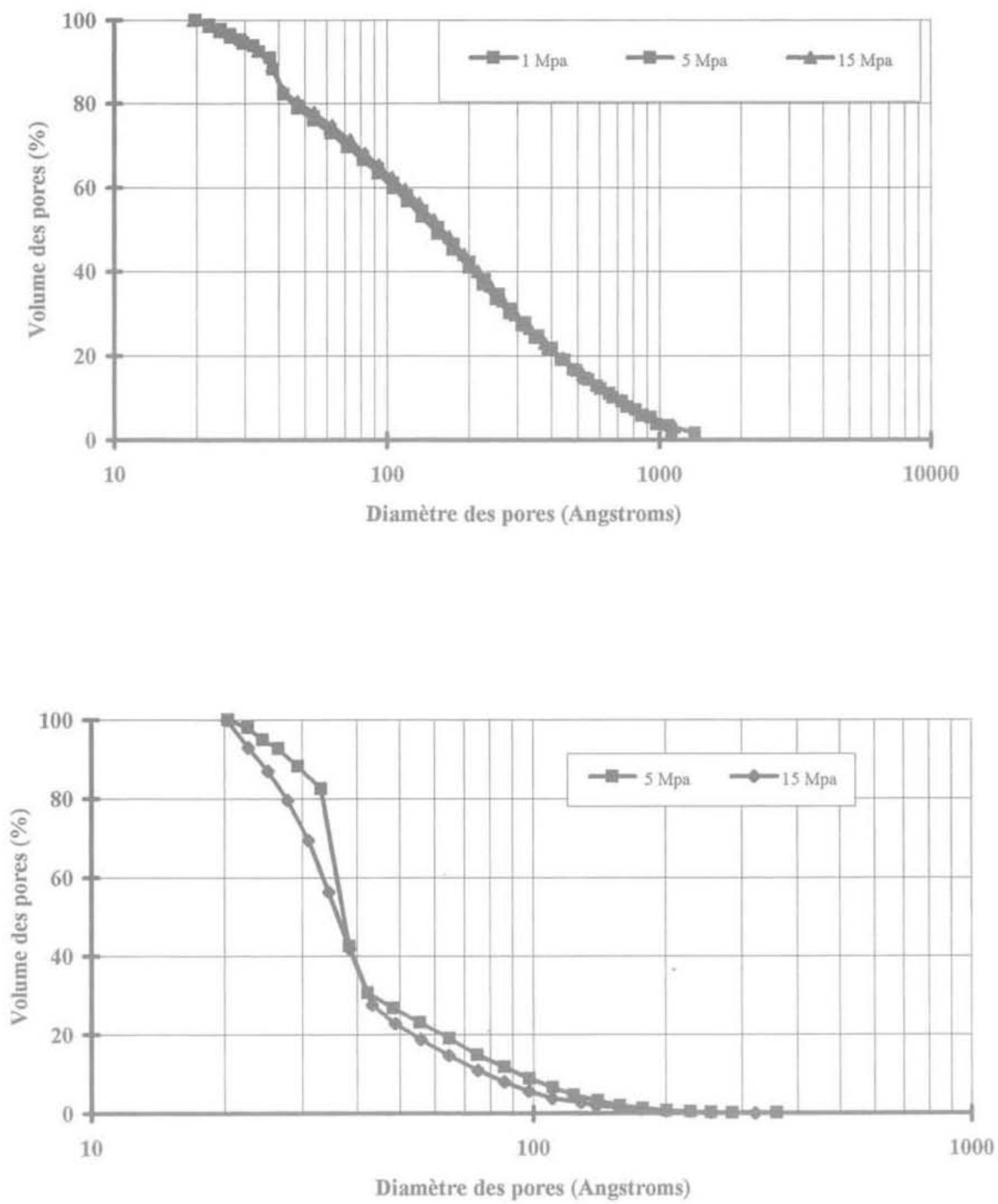

FIG. - Microporosité de l'argile du Bassin parisien (a) et de la smectite (b), Micro-porosity of the Bassin parisien clays (a) and of the smectite (b).

siment confondues, quelles que soient les pressions de consolidation;

- la pression de consolidation affecte les macroporosités de façon distincte suivant le type d'argile (Fig. 7). Si pour l'argile du Bassin parisien, nous remarquons que les courbes de distribution porale montrent une diminution du diamètre moyen des pores avec l'augmentation de la pression de consolidation, il n'en est pas de même pour la smectite. En effet, les distributions porales à 5 et $15 \mathrm{MPa}$ sont quasiment identiques. Cette différence avec l'argile du Bassin parisien est due au fait que la smectite est une argile plus gonflante,

En conclusion, nous pouvons dire que :

- l'augmentation de la pression de consolidation affecte les macropores (pores dont le diamètre est supérieur à $100 \AA$ ), mais ne modifie pas les micropores:

- l'étude de la macroporosité permet de différencier les argiles gonflantes des argiles non gonflantes. En effet, les déformations des macropores après un cycle de charge-décharge sont quasiment réversibles dans le cas des argiles gonflantes alors qu'elles sont irréversibles dans le cas des argiles non gonflantes. 
Cas de l'argile du bassin parisien

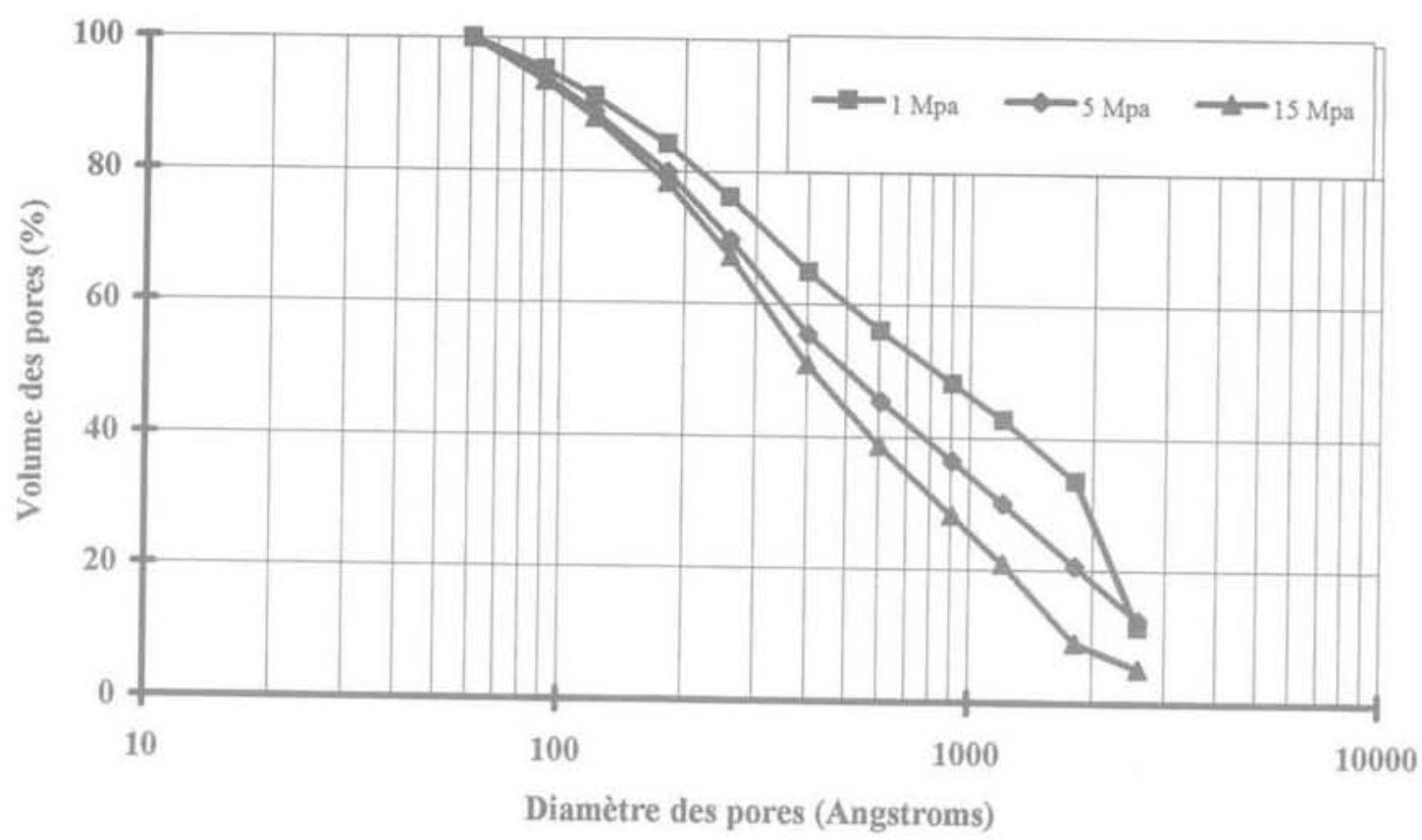

Cas de la smectite

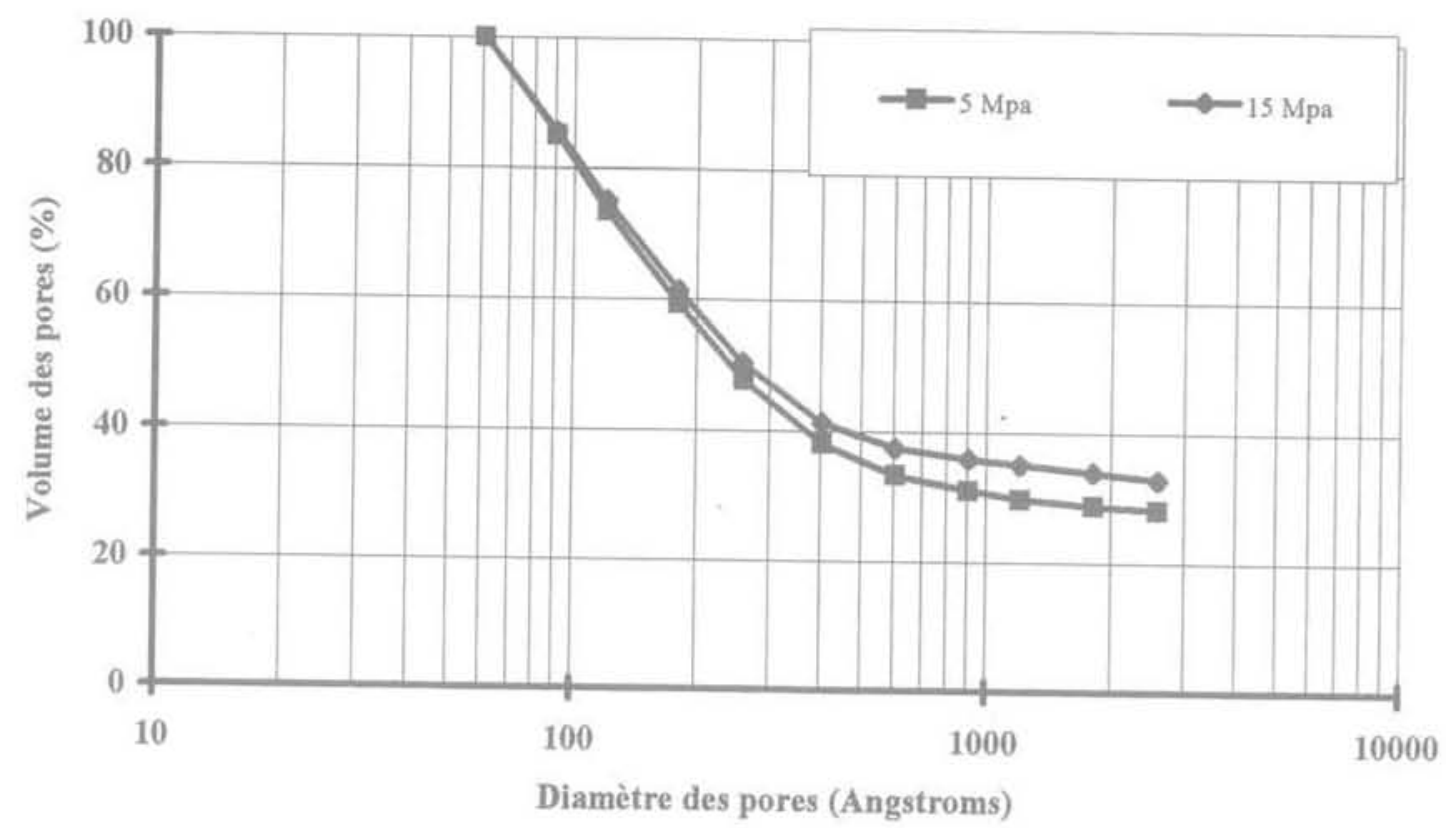

FlQ.7 Macroporosité de l'argile du Bassin parisien (a) et de la smectite (b). Macro-porosity of the Bassin parisien clay (a) and of the smectite (b).

\section{Comportement mécanique sur chemin triaxial non drainé}

L'essai est réalisé en trois étapes : deux étapes de consolidation isotrope et une étape de cisaillement. La boue est d'abord faiblement compactée à $0,5 \mathrm{MPa}$ de manière isotrope dans un consolidomètre. Les échan- tillons sont alors découpés avec un élancement de 1 (diamètre $=$ hauteur $=65 \mathrm{~mm}$ ), puis placés dans la cellule trixiale. Une deuxième consolidation isotrope est alors réalisée. Une fois cette deuxième étape terminée, les conduits de drainage sont fermés avant de procéder au cisaillement de l'échantillon. Cette phase se poursuit jusqu'à la rupture de l'échantillon. L'enregistrement des résultats « bruts ») de l'essai se fait grâce à un système d'acquisition, en vue d'un traitement ultérieur. 


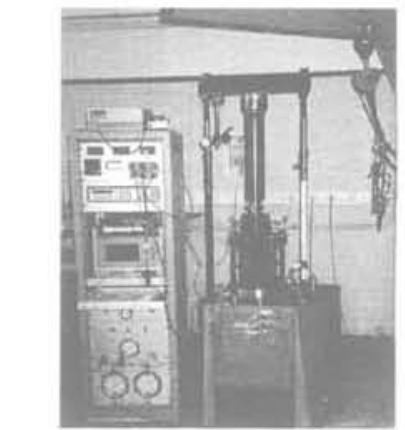

1 Echantilition

2 Chumbre de confinemern

3 Collier de mataie du diameire

4 Capteait de deplacerriemt ineme

5 Commmication entre les ehamber

6 Chambe de compenanice

7 Pistoc d'ernuement

8 Preme de ensuencent

9 Ciraiti hydraifipu?

10 Aecumulateur huile;

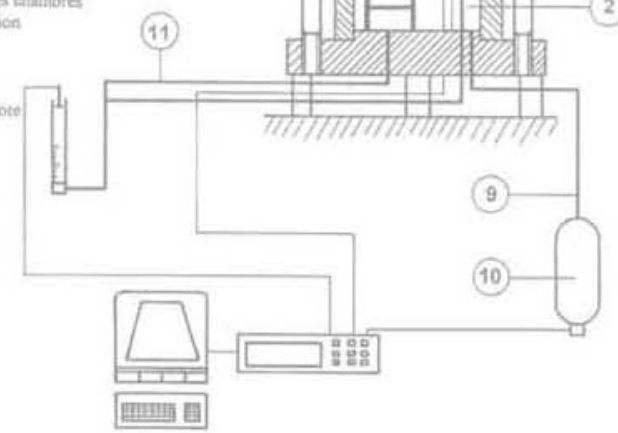

Fig. 8 Coupe schématique de la cellule triaxiale à haute pression.

Schematic section of high pressure triaxial cell.

\subsection{1}

\section{Résultats obtenus et interprétation}

Nous avons réalisé trois essais triaxiaux de cornpression non drainés normalement consolidés, à des pressions de confinement de 5 à $10 \mathrm{MPa}$ sur la kaolinite et de $5 \mathrm{MPa}$ sur la smectite. Les courbes présentées pour chaque essai illustrent :

- la variation du déviateur de contrainte (q) en fonction de la déformation axiale $\left(\varepsilon_{i}\right)$;

- la variation de la pression interstitielle $(\Delta \mathrm{u})$ en fonction de la déformation axiale $\left(\varepsilon_{1}\right)$;

- la variation du déviateur de contraintes (q) en fonction de la pression moyenne effective ( $\left.p^{\prime}\right)$.

Les résultats des deux essais réalisés sur une kaolinite normalement consolidée aux pressions de confinement de 5 et $10 \mathrm{MPa}$ (Fig. 9), permettent de faire les observations suivantes :

- les courbes représentant la variation du déviateur de contraintes en fonction de la déformation axiale montrent un léger pic correspondant à la valeur maximale du déviateur. Celui-ci survient pour des valeurs de déformations axiales à peu près identiques, dans le cas des essais considérés. Puis la valeur du déviateur chute légèrement pour commencer à se stabiliser et à tendre vers un palier à partir d'une valeur de déformation axiale correspondant à $12 \%$ :

- les courbes représentant la variation de la pression interstitielle en fonction de la déformation axiale montrent que la pression interstitielle continue à évoluer légèrement contrairement au pic observé dans le plan $\left(\mathrm{q}, \varepsilon_{1}\right)$;

- dans le plan (q, p'), le chemin des contraintes effectives, après le pic correspondant au déviateur maximum, se déplace sur la droite de plasticité parfaite, caractérisée par la pente $M=q / p^{\prime}$.

Les caractéristiques mécaniques de la kaolinite sont les suivantes:

- angle de frottement $\left(\varphi^{\prime}\right): 14,8$ degrés ;

$-\mathrm{M}=0,5$.

La figure 10 illustre les résultats des essais effectués à une pression de confinement de $5 \mathrm{MPa}$ sur la kaolinite et la smectite. Les constatations suivantes ont été faites :

- de légers plans de cisaillement ont été observés après l'essai sur l'échantillon de kaolinite mais pas sur l'échantillon de smectite ;

- le pic observé dans le plan $\left(q, \varepsilon_{1}\right)$ dans le cas de la kaolinite n'a pas été observé dans le cas de la smectite. De plus, le palier de plasticité parfaite de la smectite se confond avec sa résistance maximale;

- le déviateur à la rupture de la kaolinite est plus important que celui de la smectite. Ils sont en effet respectivement égaux à $1,73 \mathrm{MPa}$ et $0,77 \mathrm{MPa}$. Par contre, la déformation à la rupture de la kaolinite est plus faible que celle de la smectite, puisqu'elles sont respectivement égales à $9,3 \%$ et $11,8 \%$.

\subsection{5}

\section{Déformation des textures après des essais triaxiaux}

La figure 11 présente l'évolution de la porosité sur des échantillons de kaolinite après des essais triaxiaux réalisés avec des confinements de $2,5 \mathrm{MPa}(\mathrm{OCR}=2)$ et $5 \mathrm{MPa}(\mathrm{OCR}=1)$ et les compare à l'échantillon de kaolinite consolidée à $5 \mathrm{MPa}$. Ces résultats montrent que le cisaillement entraîne des déformations de la texture, puisque la distribution porale est différente avant et après le cisaillement. Les compressions triaxiales produisent un compactage des mésopores sans une modification significative de la distribution du diamètre des pores.

\section{3}

\section{Comportement thermo-mécanique des roches argileuses remaniées, saturées}

\section{1}

\section{Comportement thermo-mécanique sur che- min œdométrique}

Les essais œedornétriques en température sont réalisés en utilisant le dispositif œdométrique (Fig. 1). La cellule est introduite à l'intérieur de l'étuve autorégulée puis raccordée au circuit de saturation et de chargement hydraulique.

L'effet de la température sur le comportement mécanique des argiles remaniées, saturées et fortement consolidées, peut être analysé, d'une part, dans le cas où celle-ci est constante, et son effet est alors associé à celui de la charge mécanique et, d'autre part, dans le cas d'une charge mécanique maintenue constante alors que la température varie, et dans ce cas, seul l'effet de 

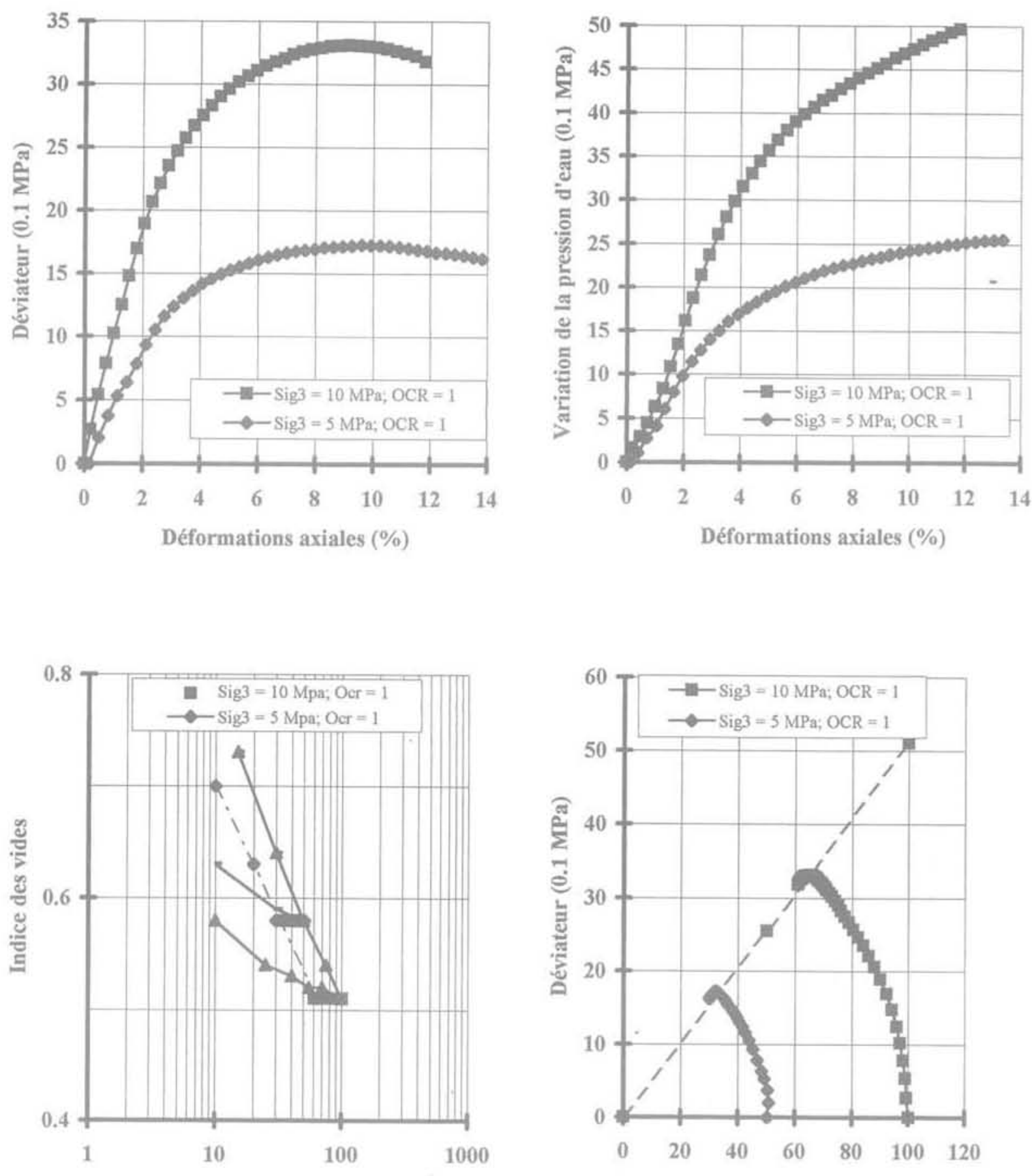

Pression moyenne effective $(0.1 \mathrm{Mpa})$

Pression moyenne effective ( $0.1 \mathrm{MPa})$

FiG.9 Essais triaxiaux non drainés sur une kaolinite normalement consolidée à des pressions de confinement de 5 et $10 \mathrm{MPa}$.

Undrained triaxial tests on normally consolidated kaolinite under a confining pressure of 5 and $10 \mathrm{MPa}$.

la dite température est étudiée. Les deux aspects de l'influence de la température correspondent respectivement aux essais isothermes et aux essais avec cycle(s) de température.

\section{1}

\section{Cas des essais isothermes}

Dans le cas de l'argile du Bassin parisien, les 62 normalement consolidé et ont débuté après que la cellule œdométrique contenant l'échantillon ait été portée pendant vingt-quatre heures à la température de l'essai $\left(20,40\right.$ et $80^{\circ} \mathrm{C}$ ) (Fig. 12). Nous observons alors qu'en charge et en décharge, les courbes tracées dans le plan (e, Log p'), sont linéaires et quasiment parallèles pour les trois températures, et que l'indice des vides diminue avec la température. Nous pouvons donc en déduire que, d'une part, les variations de l'indice des vides pour les trois températures ne sont dues qu'à la densification initiale du squelette solide et que, d'autre part, la température ne modifie 

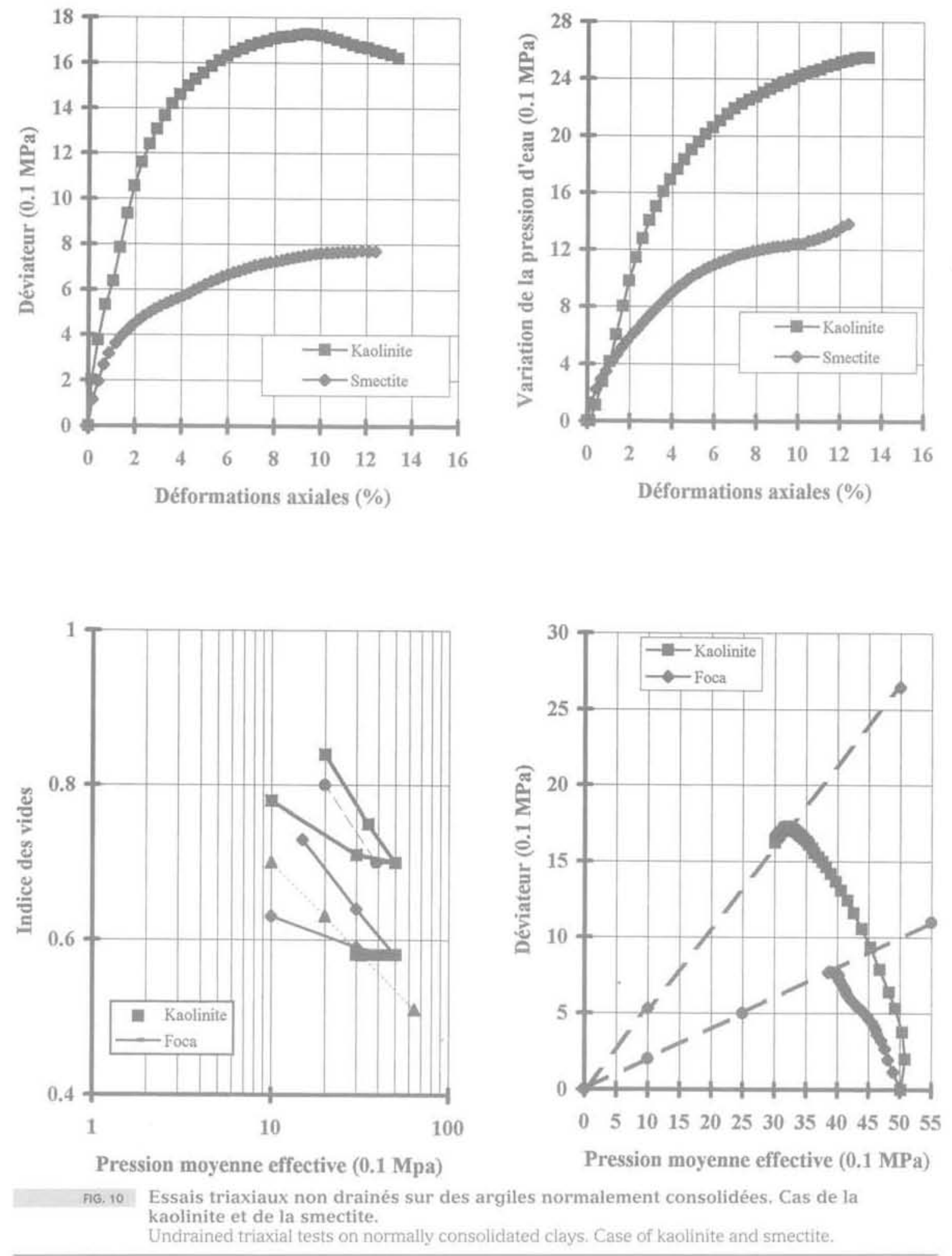

pas la compressibilité plastique. Par contre, dans le cas de la kaolinite et de la smectite, les argiles ont été consolidées sous $1,5 \mathrm{MPa}$, et le chauffage à $60^{\circ} \mathrm{C}$ a commencé sous une charge de $0,5 \mathrm{MPa}$. Les figures 13 et 14 comparent les consolidations à 20 et $60^{\circ} \mathrm{C}$, respectivement dans les cas de la kaolinite et de la smectite.

Ces deux argiles présentent des pentes de charge indépendantes de la température. Par contre, l'aug- mentation de la température a, d'une part, provoqué une densification du matériau identique à celle de l'argile du Bassin parisien et, d'autre part, diminué dans le domaine élastique et donc sa pression de consolidation. Par ailleurs, la pente de décharge de la kaolinite est indépendante de la température alors que celle de la smectite diminue quand la température augmente. L'augmentation de la température semble donc diminuer les aptitudes de gonflement de la smectite. 

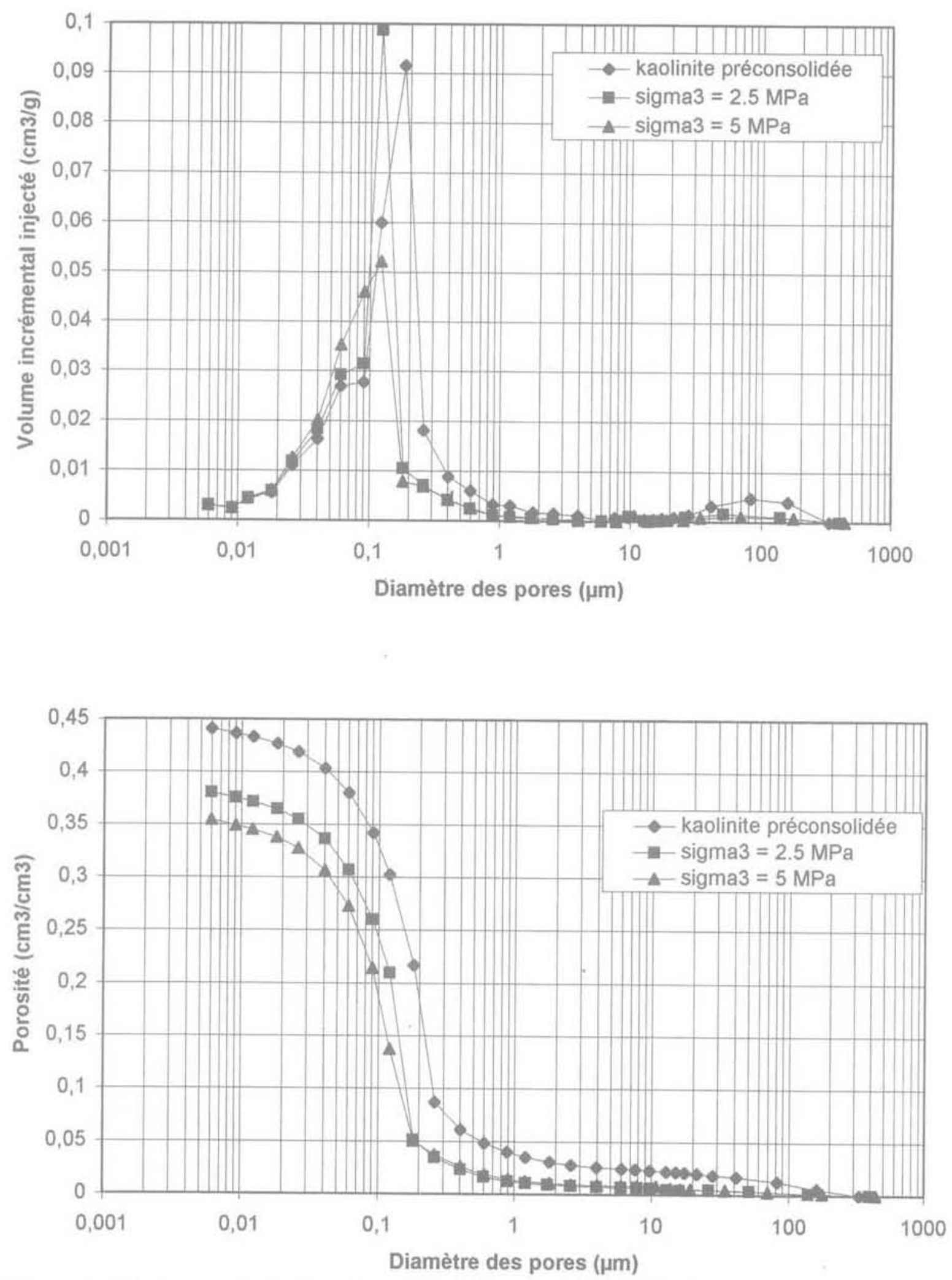

FiG. 11 Macroporosité d'échantillons de kaolinite consolidés sous 5 MPa et après cisaillement sous des pressions de confinement de 2,5 et $5 \mathrm{MPa}$.

Macro-porosity of kaolinite samples consolidated under $5 \mathrm{MPa}$ and after shearing at a confining pressure of 2,5 and $5 \mathrm{MPa}$. 

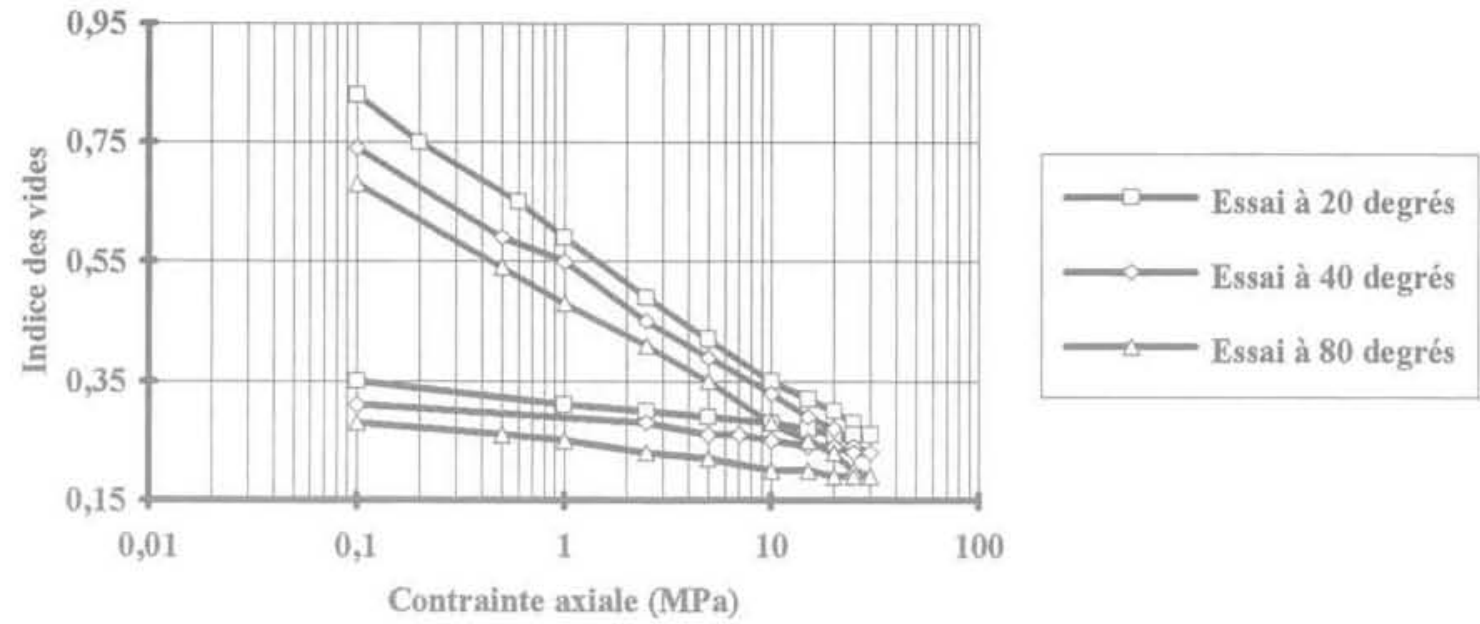

FIG. 12 Essais cedométriques isothermes sur l'argile du Bassin parisien. Isothermal oedometric tests on Bassin parisien clay.

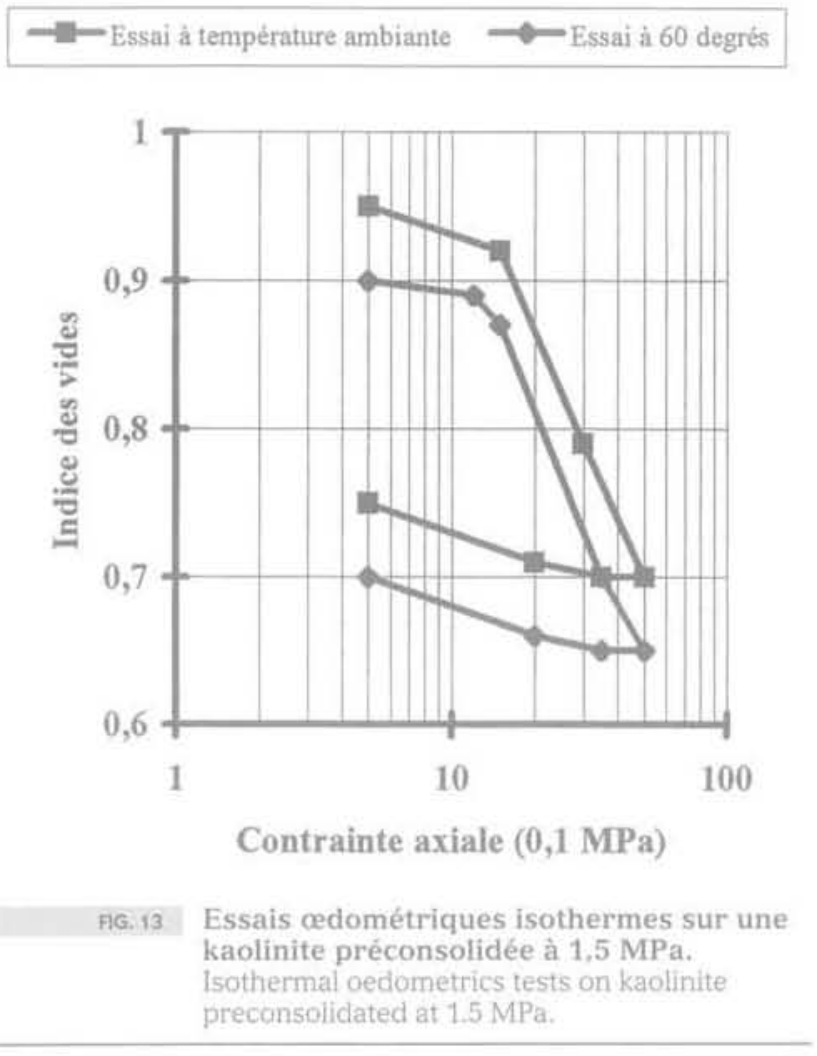

\section{3iflor}

Cas des essais avec cycle(s) thermique(s)

Ils permettent d'examiner, pour une pression de chargement fixe, la variation des déformations volumiques d'un sol aussi bien à l'état normalement consolidé qưá l'état surconsolidé. Les résultats des différents essais avec cycle(s) thermique(s) sont présentés sur les figures $15,16,17,18$ et 19 .

\section{- Cycles thermiques à l'état normalement conso- lidé}

Des essais œdométriques ont été menés sur la smectite et sur les argiles de Boom et du Bassin pari-

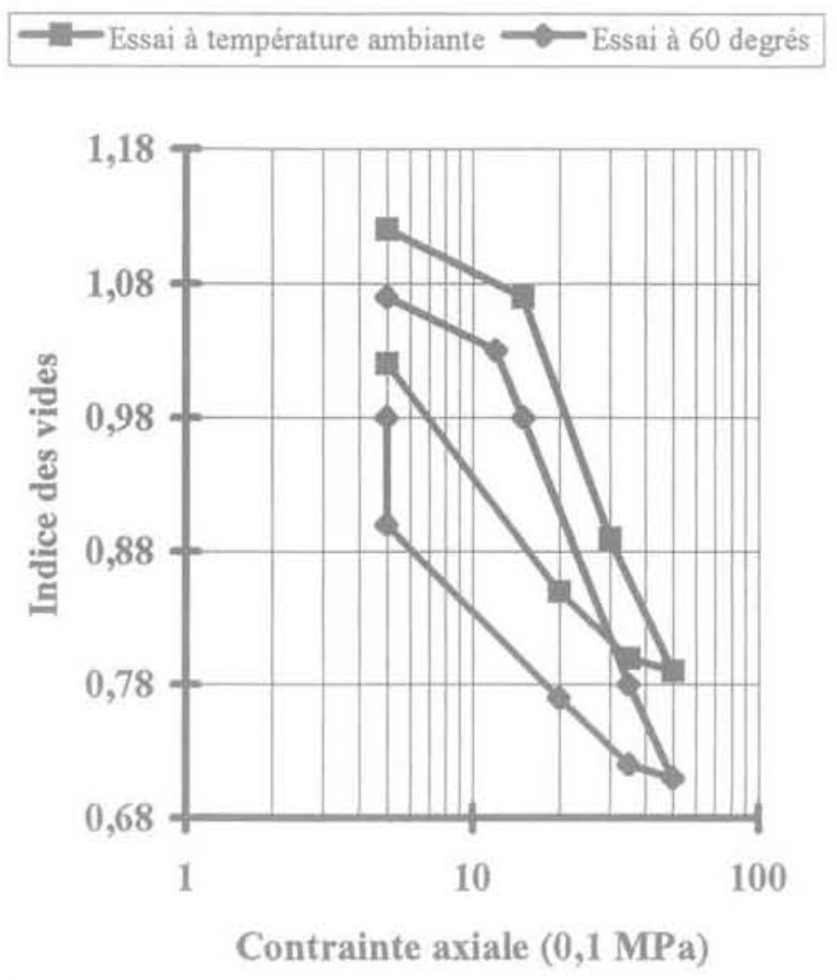

FIG.14 Essais cedométriques isothermes sur une smectite préconsolidée à 1,5 $\mathrm{MPa}$. Isothermal oedometric tests on smectite preconsolidated at $1.5 \mathrm{MPa}$.

sien. Lors de la charge, un cycle thermique $20-80-20^{\circ} \mathrm{C}$ a été appliqué sous une charge mécanique constante respectivement égale à $2,5 \mathrm{MPa}$ et $5 \mathrm{MPa}$. Les figures 15,16 et 17 présentent les résultats. L'application d'un cycle thermique à l'état normalement consolidé entraîne donc une diminution irréversible de l'indice des vides et de la pente de charge.

\section{- Cycle(s) thermique(s) à l'état surconsolidé}

Nous avons réalisé le même cycle thermique à l'état surconsolidé $(O C R=10)$ sur la smectite et l'argile du Bassin parisien. Dans les deux cas le cycle de température produit un gonflement irréversible du matériau (Fig. 18, 19). 


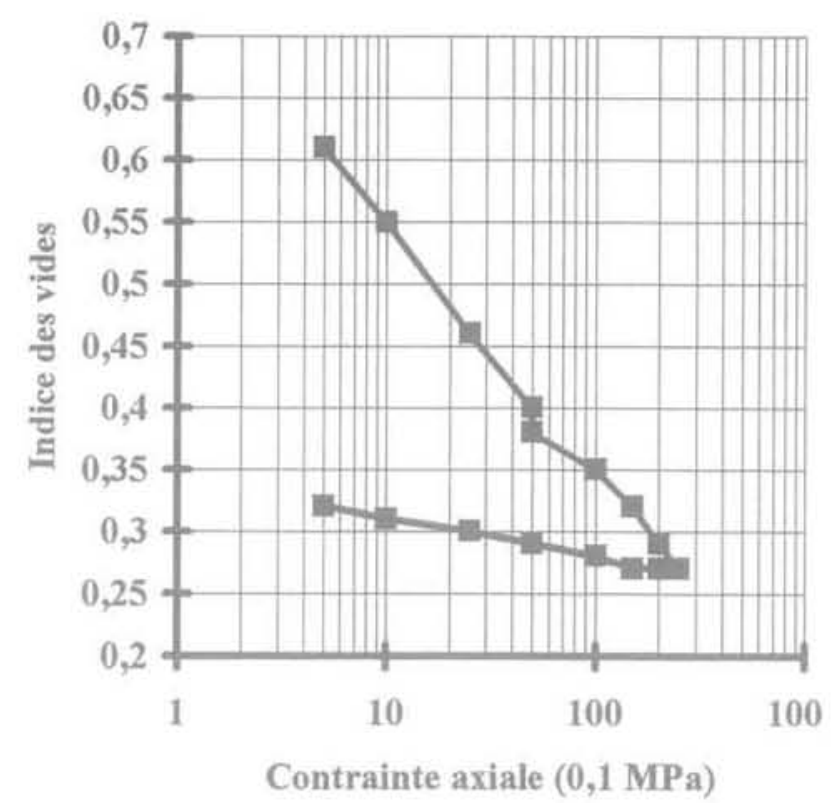

-Cycle thermique (20-80-20) à OCR $=1$ sur une argile du bassin parisien

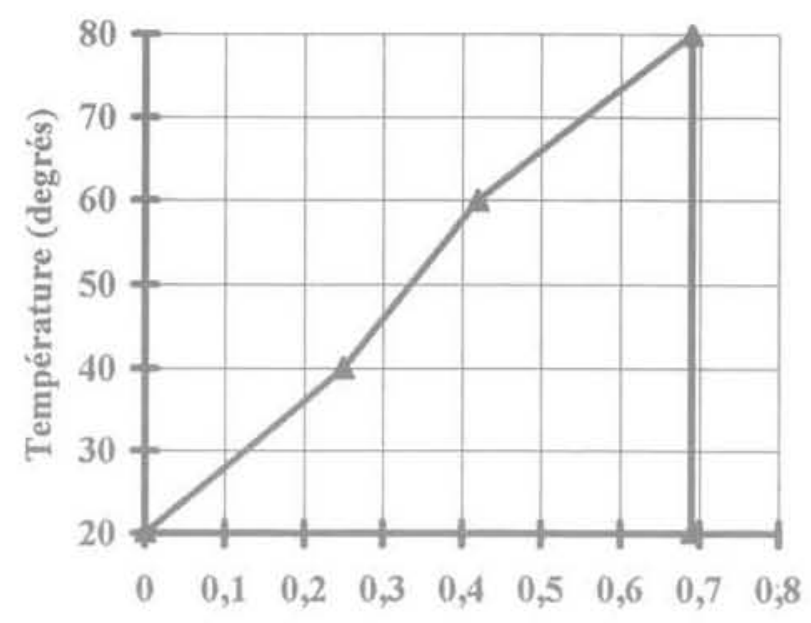

Variations des déformations volumiques

(\%)

FIG,15 Essai cedométrique avec cycle de température sur une argile du Bassin parisien.

Dedometric test with cycle of temperature on Bassin parisien clay.

- Étude de l'anisotropie des déformations sous sollicitations thermiques

Des échantillons de smectite et de kaolinite ont été fabriqués par compactage œdométrique à partir d'une boue sous une contrainte de $15 \mathrm{MPa}$. Les échantillons ont alors subi des cycles thermiques dans une enceinte à une humidité relative de $100 \%$, afin de maintenir les échantillons saturés. Les déformations axiales et radiales de l'échantillon sont mesurées à la fin de

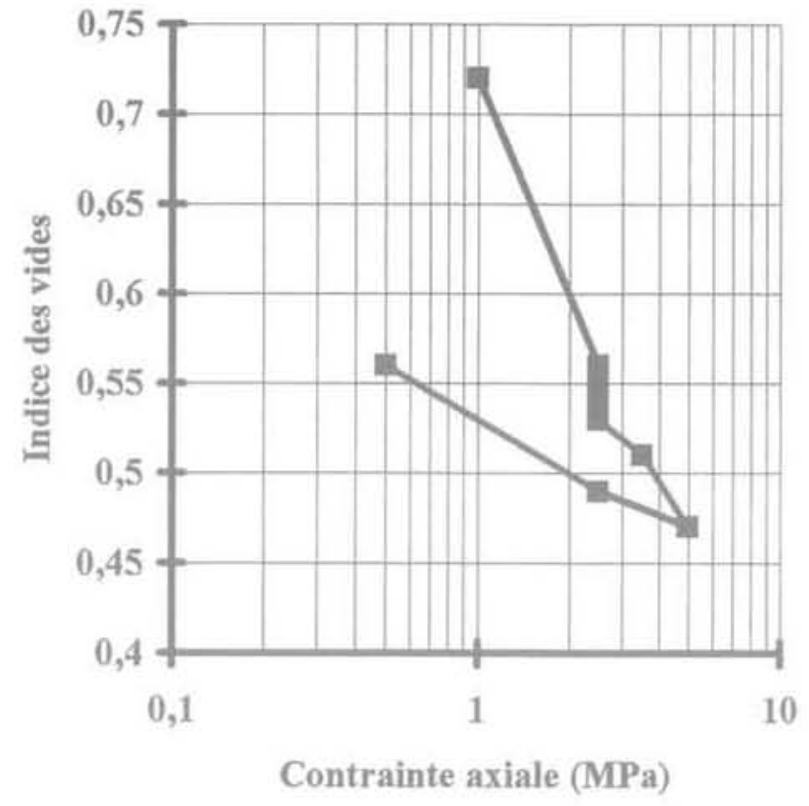

- Cycle thermique (20-80-20) á OCR $=1$ sur l'argile de Boom

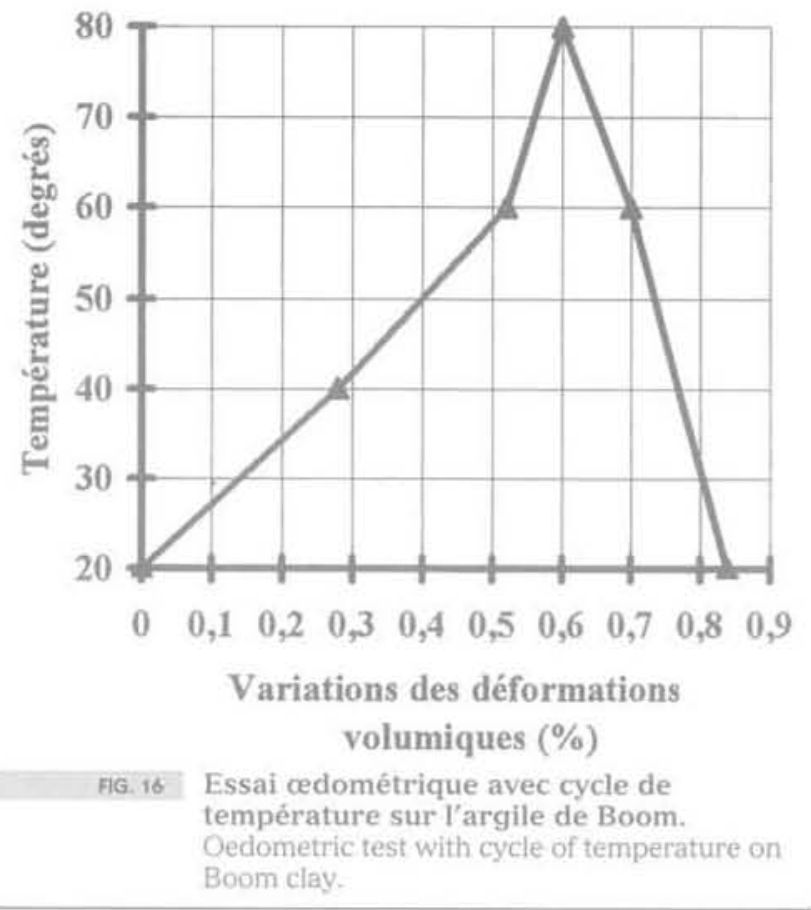

pectivement présentés sur les figures 20 (a, b et c) et 21 (a, b et c). Ces résultats montrent que :

- la courbe de variation des déformations axiales de l'échantillon a la même forme que celles obtenues précédemment pour des échantillons surconsolidés ;

- les courbes de variation des déformations axiales et radiales de l'échantillon ont la même forme. Cependant, les déformations axiales sont beaucoup plus importantes que les déformations radiales. Les déformations introduites lors d'un cycle thermique sont donc anisotropes. 


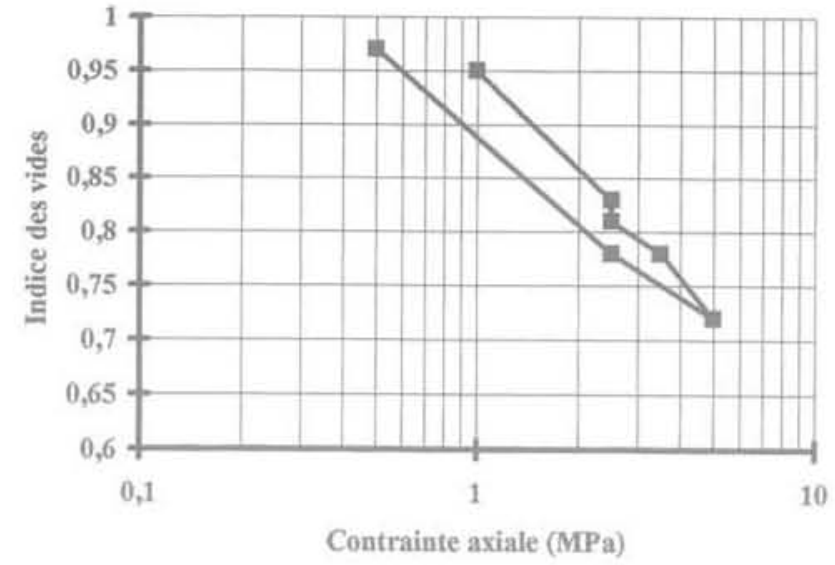

-Cycle thermique $(20-80-20)$ à $O C R=1$ sur une smectite

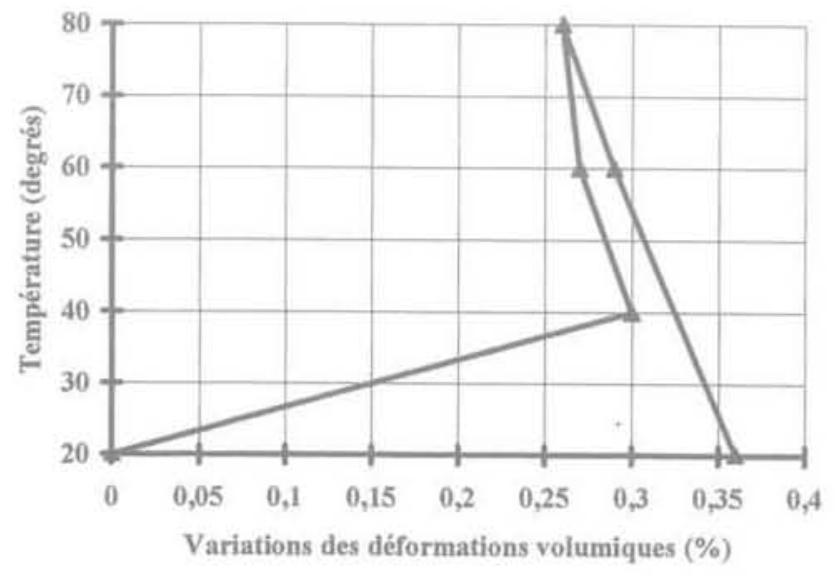

F10.17 Essai codométrique avec cycle de température sur une smectite. Oedometric test with cycle of temperature on smectite.

\section{Comportement thermo-mécanique sur chemin triaxial non drainé}

Un seul essai triaxal isotherme a été réalisé sur la kaolinite. Cet essai dans sa mise en œuvre a été analogue à un essai à température ambiante. Le chauffage du matériau a débuté entre la deuxième consolidation isotrope et le cisaillement. Les résultats des essais triaxiaux isotropes non drainés sur une kaolinite remaniée, saturée et normalement consolidée à $5 \mathrm{MPa}$, réalisés à une température de $40^{\circ} \mathrm{C}$ et à température ambiante sont présentés sur la figure 22.

Dans le plan $\left(q, \varepsilon_{1}\right)$, la courbe correspondant à l'essai réalisé à une température de $40^{\circ} \mathrm{C}$, montre une plus grande déformabilité du matériau, comparativement à l'essai à température ambiante. La rupture pour les essais à $40^{\circ} \mathrm{C}$ et à température ambiante se produit pour environ la même valeur de déformation axiale, mais pour un déviateur de contraintes plus faible dans le cas de l'essai à $40^{\circ} \mathrm{C}$. La phase post-rupture se caractérise pour l'essai à température ambiante par une légère chute du déviateur et par sa stabilisation à partir

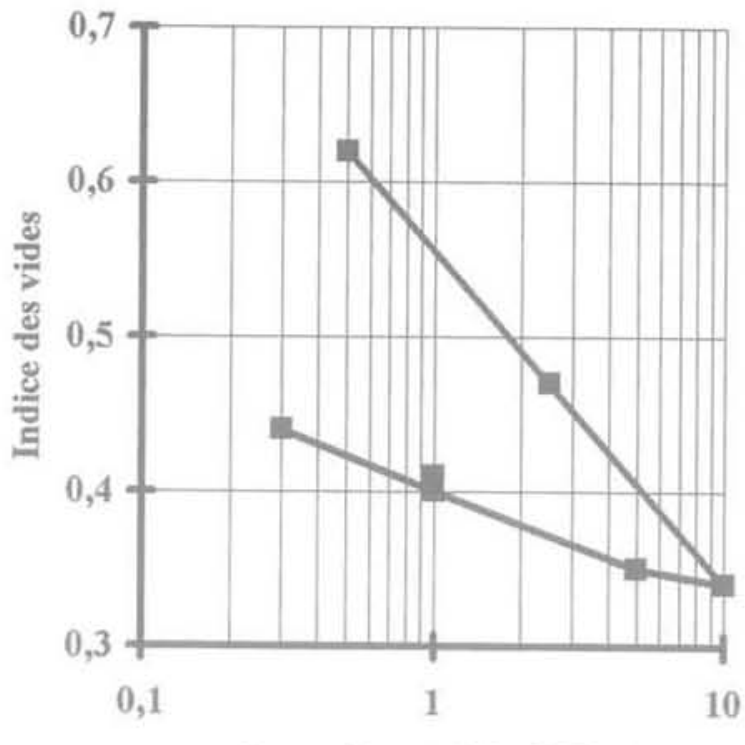

Contrainte axiale (MPa)
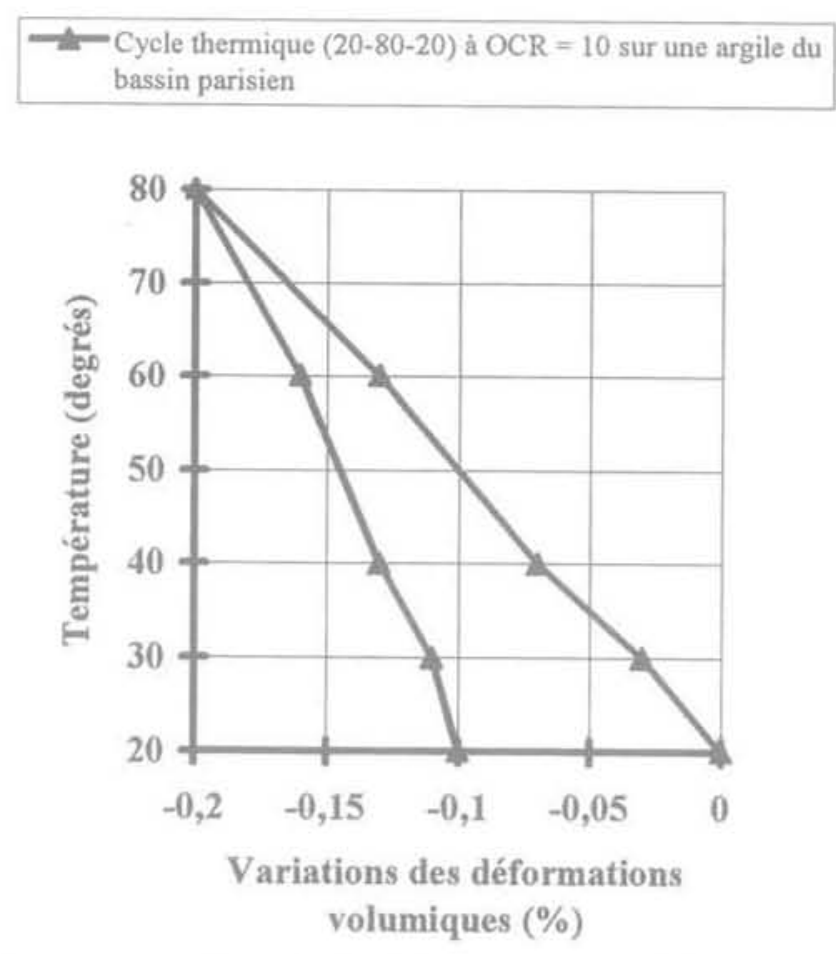

f6. 18 Essai cedométrique avec cycle de température sur l'argile du Bassin parisien.

Oedometric test with cycle of temperature on Bassin parisien clay.

de $12 \%$ de déformations axiales. En revanche, dans le cas de l'essai à $40^{\circ} \mathrm{C}$, on note une chute significative du déviateur, comparable à celle observée dans le cas des sols surconsolidés. Un début de stabilisation du déviateur est perceptible à partir d'environ $12 \%$ de déformations axiales.

Par ailleurs, l'étude des caractéristiques de la kaolinite, issues des essais triaxiaux montrent que la température influe sur ses propriétés mécaniques (tableau II). 


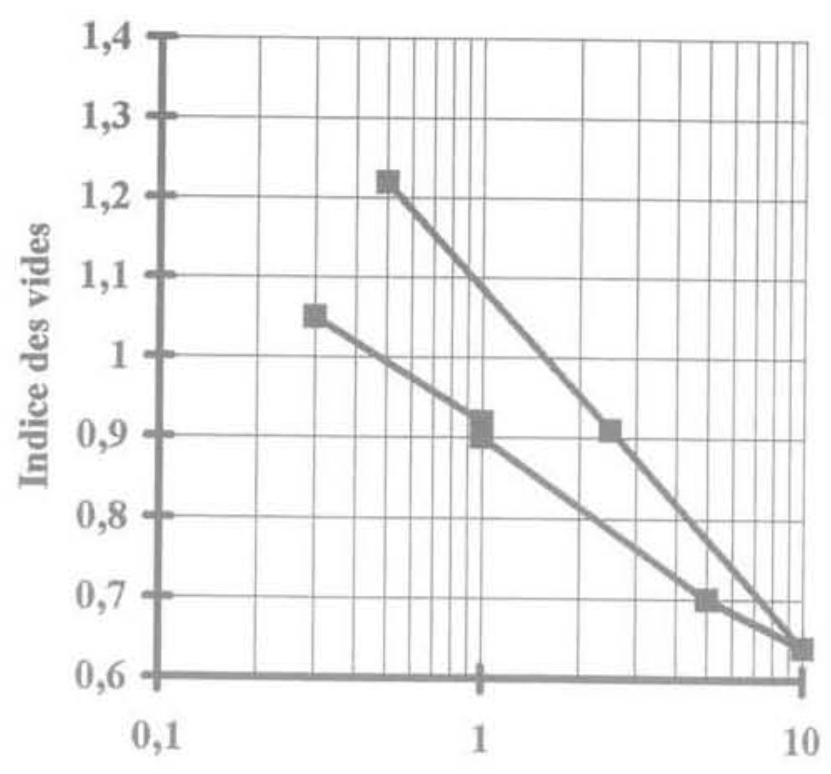

Contrainte axiale (MPa)
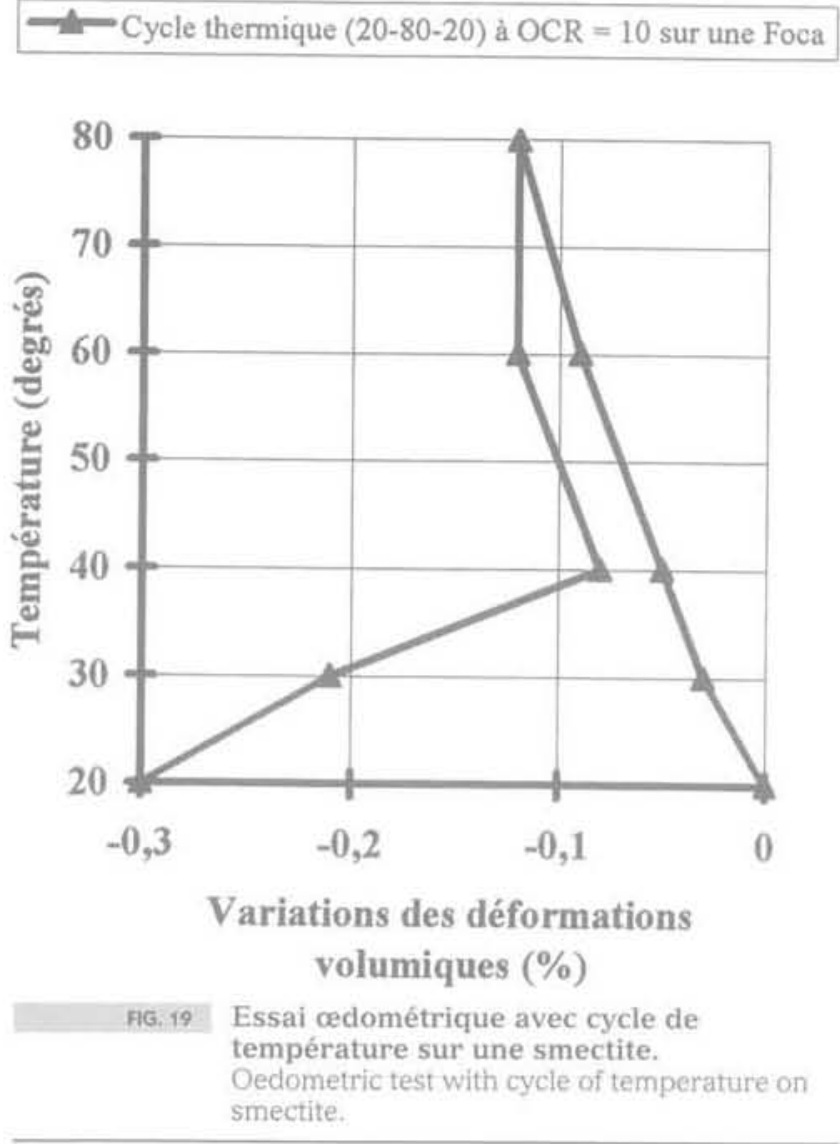

\section{3}

\section{Déformations des textures des roches argileuses sous l'effet thermique}

Les figures 23 et 24 montrent les résultats obtenus

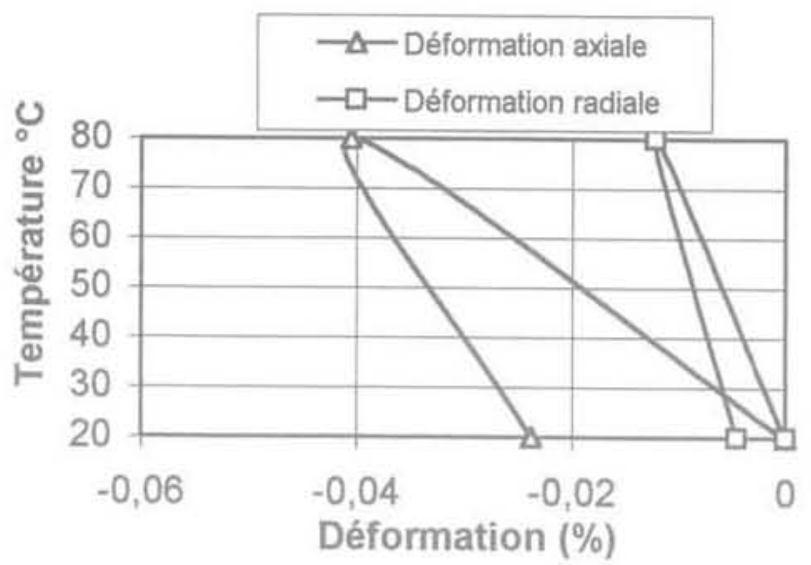

(a) 20 - 80 - 20 degrés

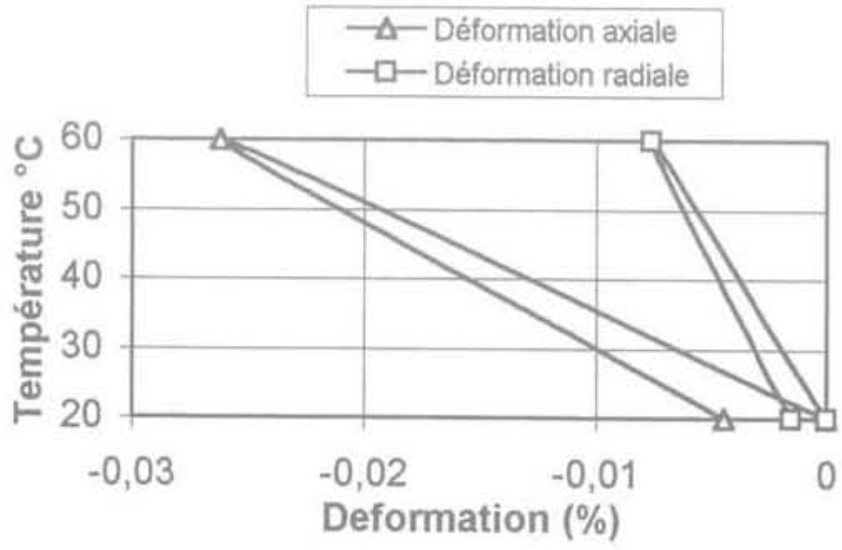

(b) $20-60-20$ degrés

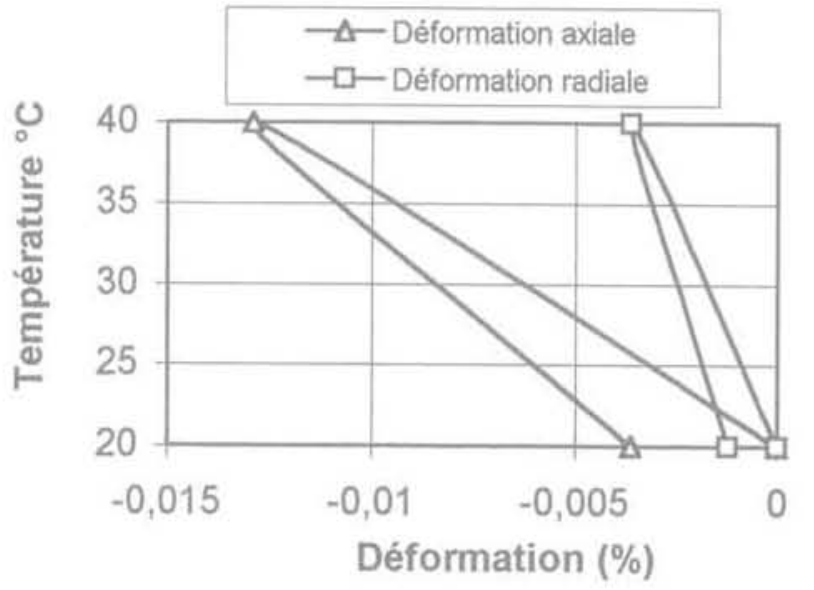

(c) $20-40$ - 20 degrés

FG. 20 Variations des déformations axiales et radiales sur un échantillon de smectite surconsolidée à $15 \mathrm{MPa}$ au cours d'un cycle thermique.

Axial and radial strain variations on smectite sample loaded at $15 \mathrm{MPa}$ during a thermal cycle.

kaolinite consolidée œedométriquement à $15 \mathrm{MPa}$ après différents cycles thermiques. Il apparaît que l'application de la contrainte thermique ne modifie pas la taille des pores du réseau bimodal $(600 \AA$ et $1200 \AA)$. Par contre, elle diminue le nombre de pores et donc le 


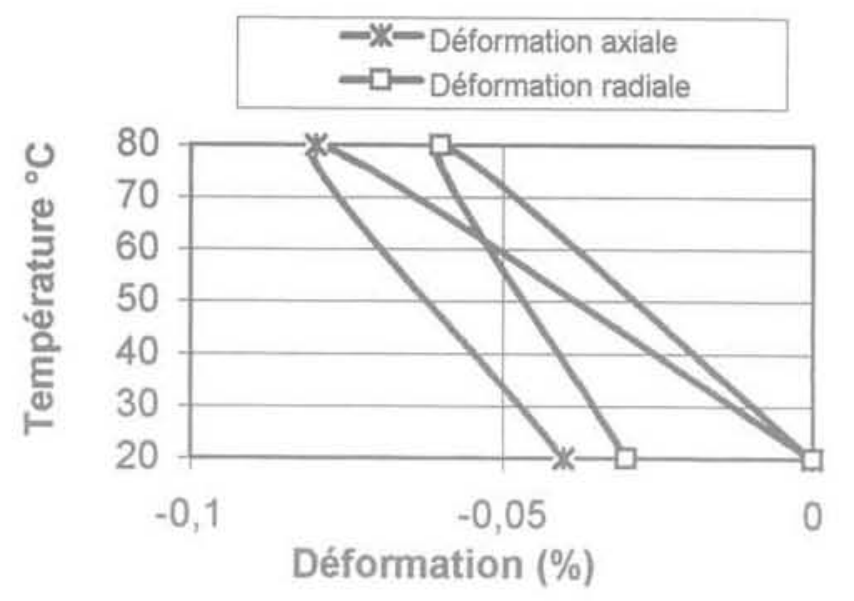

a) 20 - $80-20$ degrés

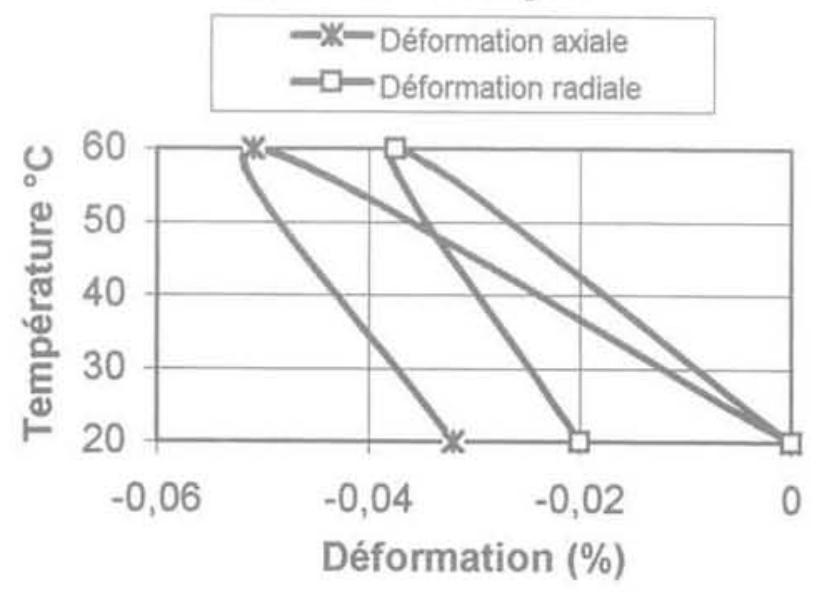

(b) $20-60-20$ degrés

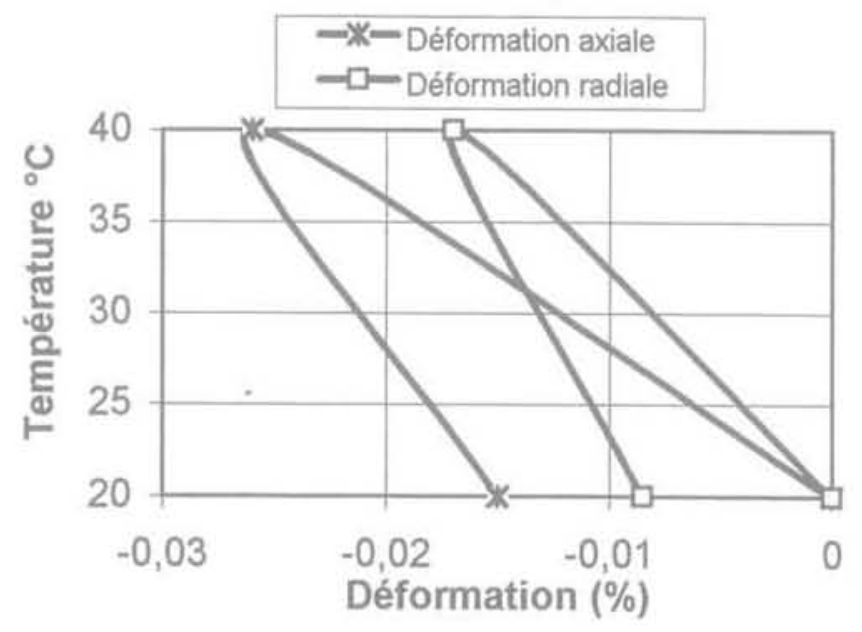

(c) 20 - 40 - 20 degrés

FG. 21 Variations des déformations axiales et radiales sur un échantillon de kaolinite surconsolidée à $15 \mathrm{MPa}$ au cours d'un cycle thermique.

Axial and radial strain variations on kaolinite sample loaded at $15 \mathrm{MPa}$ during a thermal cycle. volume cumulé total. Sur les figures 25 et 26 sont présentés les résultats d'essais de porosimétrie au mercure et de BET dans les mêmes conditions pour un échantillon de smectite. Les phénomènes observés sont les mêmes que pour la kaolinite.

\section{Comportement hydro-mécanique des roches argileuses partiellement saturées}

Le comportement hydro-mécanique des argiles partiellement saturées a été étudié sur des chemins œdométriques et triaxiaux dans lesquels les états hydrique et mécanique sont imposés. L'état hydrique est obtenu à partir du contrôle de l'humidité relative de l'air par des solutions salines saturées. Certains sels présentent en effet des propriétés spécifiques de sorption de l'eau et permettent d'imposer un potentiel de pression caractérisé par une valeur constante de l'humidité relative au-dessus de la solution. Ainsi, une solution aqueuse saturée en un sel adéquatement choisi donne un degré d'humidité relative fixe (Aldrich 1986-1987). La loi de Kelvin nous foumit alors la relation entre la succion et I'humidité relative : $s=\frac{p_{w} R T}{M} L_{n h}$. Cette technique est surtout utilisée pour déterminer les isothermes de sorption-désorption dans un domaine de pression allant de 2,7 à $360 \mathrm{MPa}$ (Daian, 1986).

\section{1}

\section{Comportement hydro-mécanique sur chemin œdométrique}

Les essais sont réalisés en utilisant un œdomètre haute pression à hygrométrie contrôlée (Robinet et al., 1997) (Fig. 27). Le principe de l'essai consiste à imposer aux limites de l'échantillon un air à humidité relative régulée par des solutions salines saturées. L'échangeur d'humidité relative est constitué d'une enceinte munie d'une entrée et d'une sortie d'air et d'un bac rempli de solution saline. Le bac est placé sur une balance pour quantifier les échanges hydriques au cours de l'essai. Un ventilateur assure la circulation de l'air à humidité relative contrôlée sous la face inférieure d'un échantillon confiné. La succion est mesurée au centre de l'échantillon par un capteur d'humidité relative.

\section{A.14}

\section{Cas des essais à succion constante}

Le comportement d'une argile gonflante, la smectite, et d'une argile non gonflante, la kaolinite, a été étudié sur des chemins œdométriques à différentes humidités relatives. Les échantillons, initialement à l'état de poudre, ont été conditionnés dans des dessiccateurs à l'humidité relative voulue jusqu'à stabilisation hydrique, puis placés dans le corps de la cellule œdométrique ventilée (Fig. 27) pour subir un cycle chargedécharge jusqu'à $10 \mathrm{MPa}$. Les résultats expérimentaux tracés dans le plan $\left(e, \log P^{\prime}\right)$ sont représentés figure 28 pour la kaolinite et figure 29 pour la smectite. Ils montrent que : 

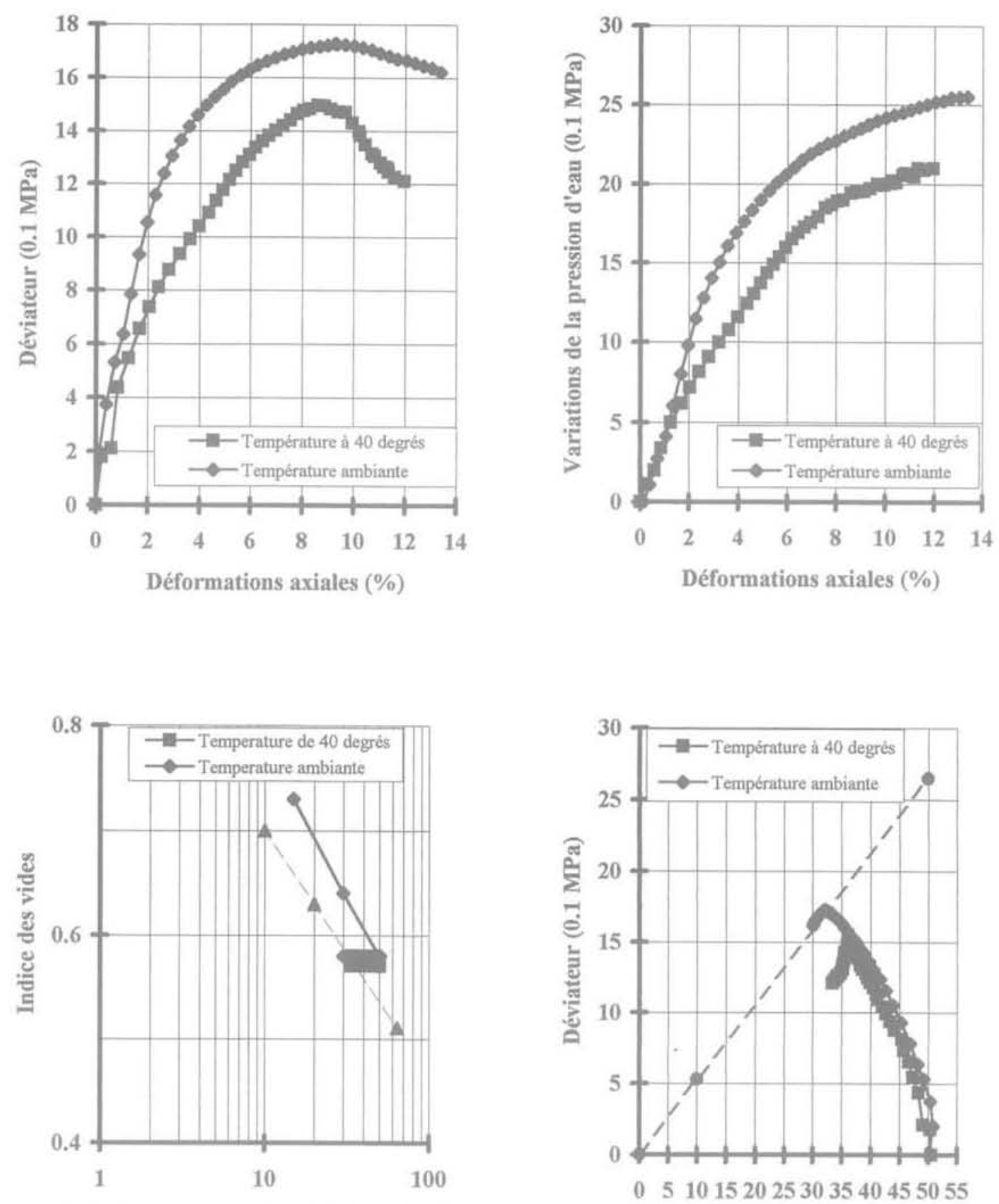

Pression moyenne effective ( $0.1 \mathrm{Mpa})$

Pression moyenne effective ( $0.1 \mathrm{MPa})$

FG. Es Essais triaxiaux isothermes non drainés sur une kaolinite normalement consolidée. Isothermal triaxial undrained tests on normally consolidated kaolinite.

- les pentes de compressibilité plastique des matériaux désaturés sont plus fortes que celles des matériaux saturés dans le cas des deux argiles :

- dans le cas de la smectite, un coude de consolidation hydrique apparaît lors du chargement, il correspond à la pression de préconsolidation du matériau. Sa valeur est d'autant plus grande que la succion l'est ;

- pour une contrainte verticale donnée l'indice des vides est d'autant plus grand que l'humidité relative imposée est faible. La succion crée donc soit une rigidification du matériau, soit une diminution de la compressibilité du sol ;
TABLEAUIII Propriétés mécaniques de la kaolinite à température ambiante et à $40^{\circ} \mathrm{C}$.

\begin{tabular}{c|c|c}
$\begin{array}{c}\text { Température } \\
\text { de l'essai }\end{array}$ & $\begin{array}{c}\text { Paramètre d'état } \\
\text { critique à la rupture }(\mathrm{M})\end{array}$ & $\begin{array}{c}\text { Angle de frottement } \\
\text { à la rupture }\left(\varphi^{\prime}\right)\end{array}$ \\
\hline $\begin{array}{c}\text { Température } \\
\text { ambiante } \\
\left(20^{\circ} \mathrm{C}\right)\end{array}$ & 0,53 & 14 \\
\hline $40^{\circ} \mathrm{C}$ & 0,36 & 9,8 \\
\hline
\end{tabular}




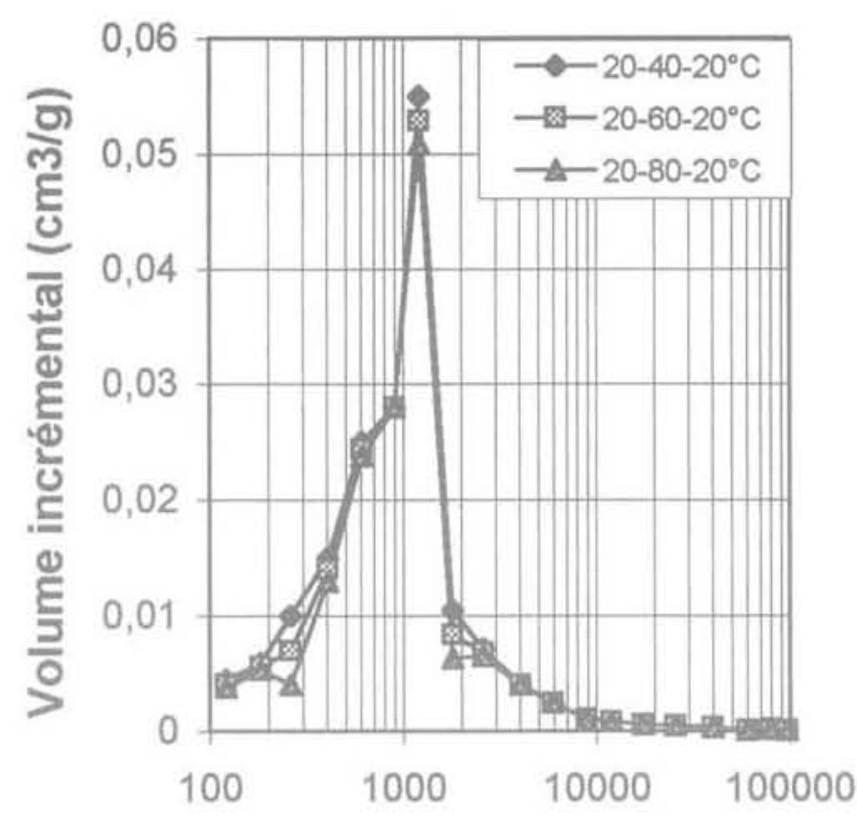

Diamètre des pores (Angströms)

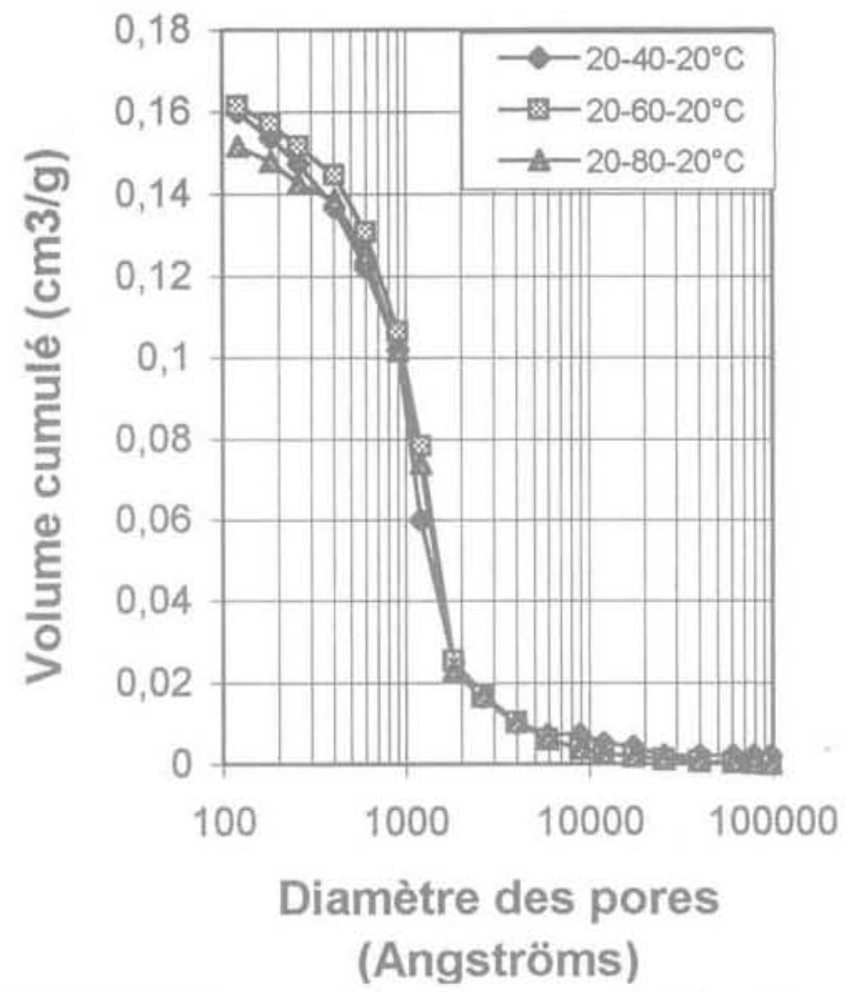

FG. 23 Porosimétrie mercure sur des échantillons de kaolinite consolidée sur un chemin œdométrique à $15 \mathrm{MPa}$ après un cycle thermique.

Macro-porosity of kaolinite samples consolidated at $15 \mathrm{MPa}$ and after thermal cycle.

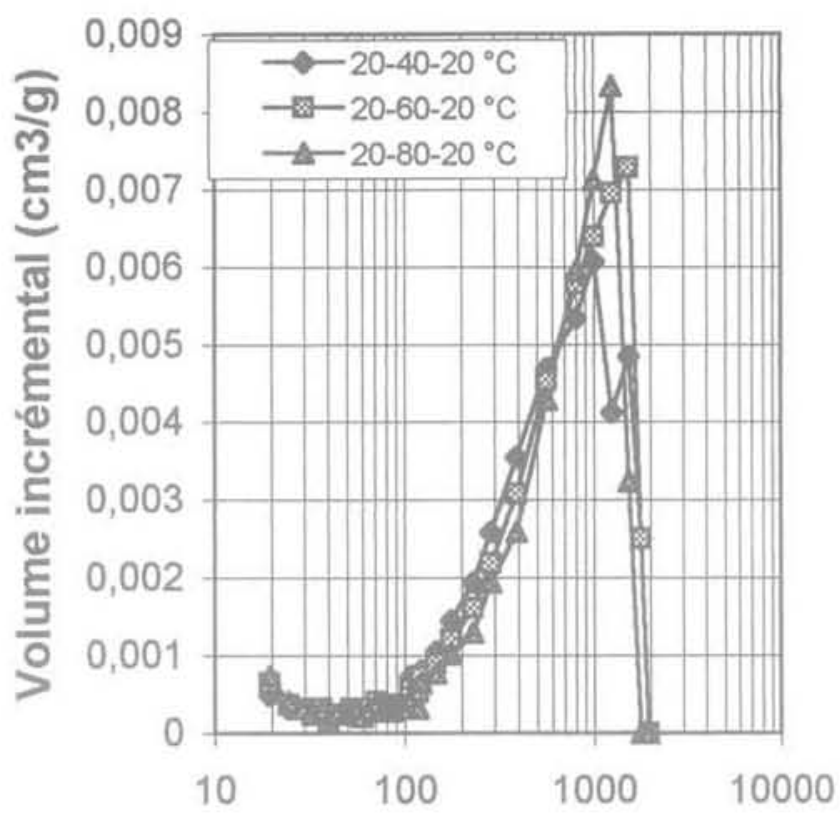

Diamètre des pores (Angströms)

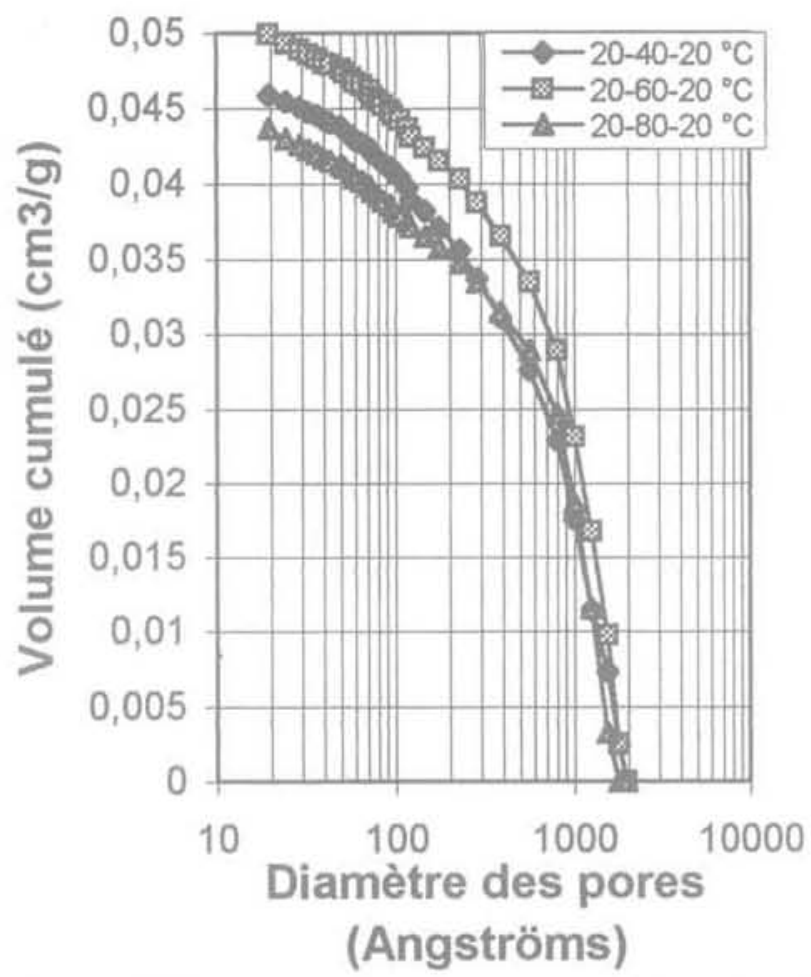

FG. 24 Désorption d'azote sur des échantillons de kaolinite consolidés sur un chemin œdométrique à $15 \mathrm{MPa}$ après un cycle thermique.

Micro-porosity of kaolinite samples consolidated at $15 \mathrm{MPa}$ and after thermal cycle. 


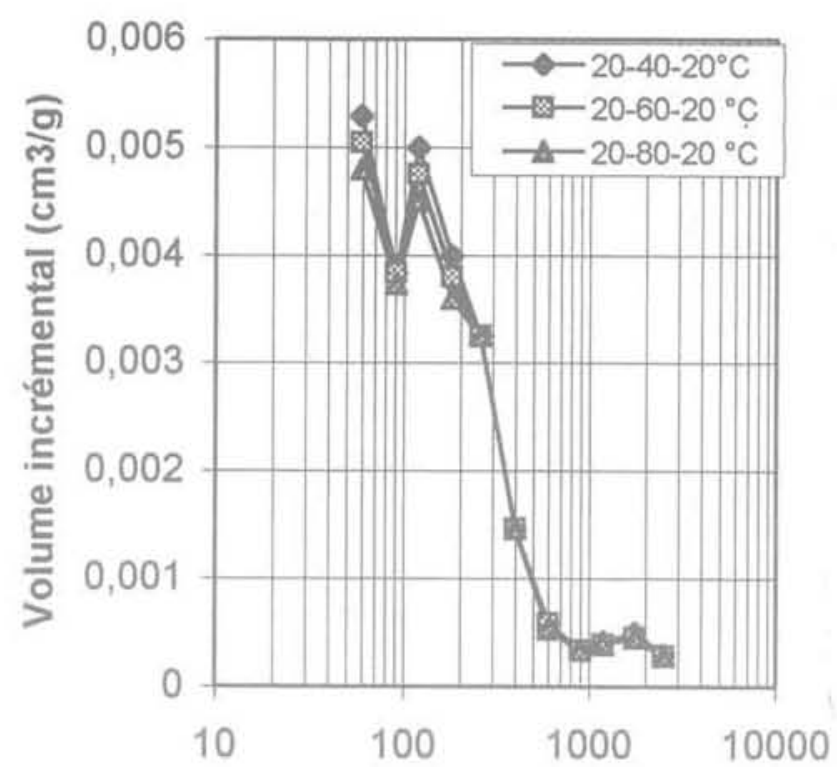

Diamètre des pores (Angströms)

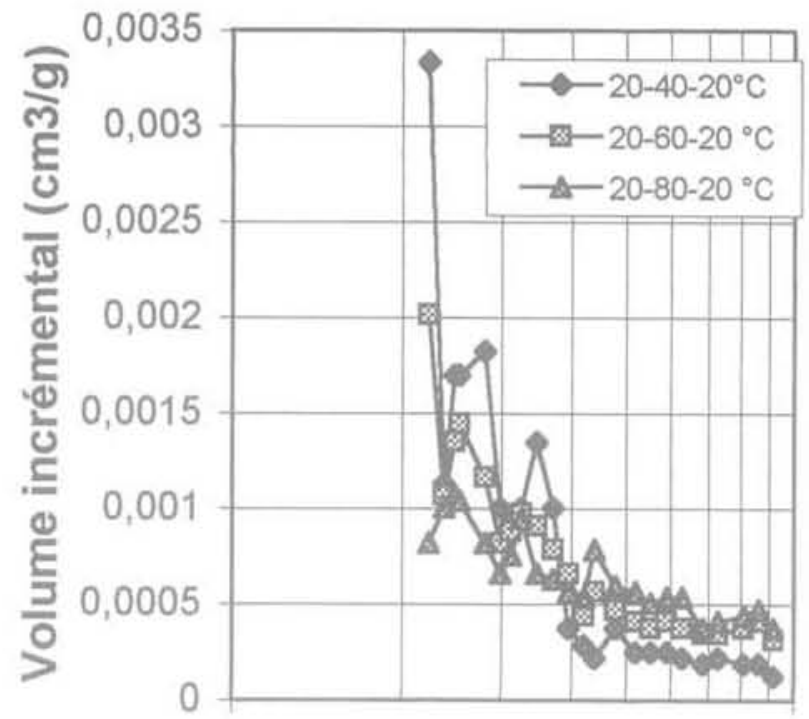

10 Diamètre des pores 100 (Angströms)

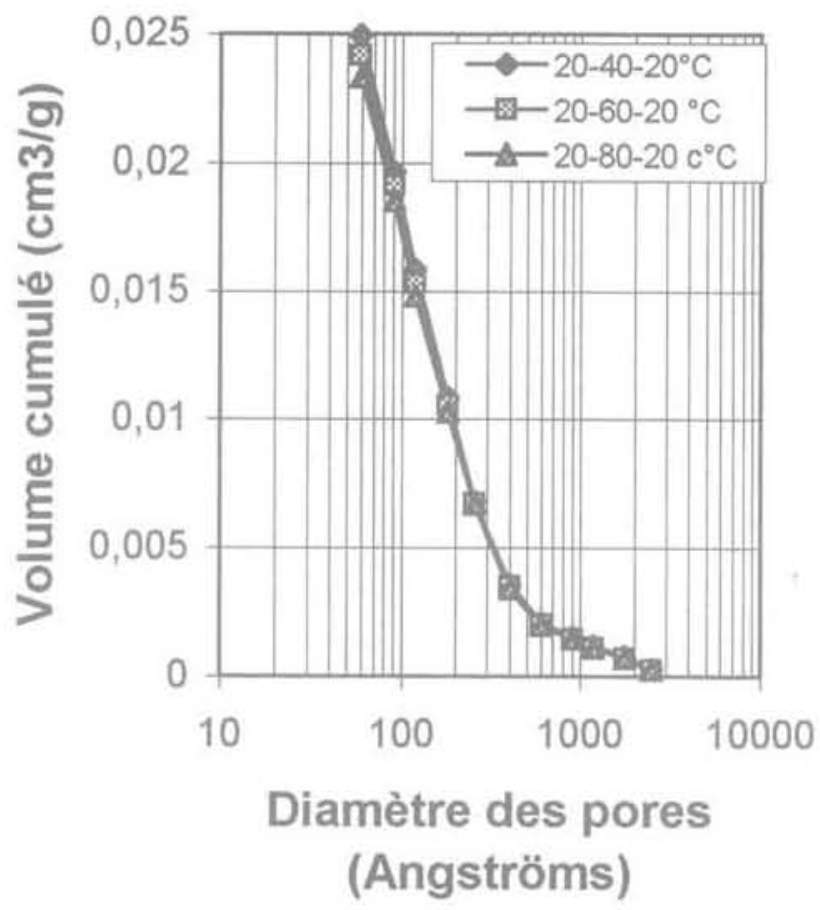

HG. 25 Porosimétrie au mercure sur des échantillons de smectite consolidée sur un chemin œdométrique à $15 \mathrm{MPa}$ après un cycle thermique.

Macro-porosity of smectite samples consolidated at $15 \mathrm{MPa}$ and after thermal cycle,

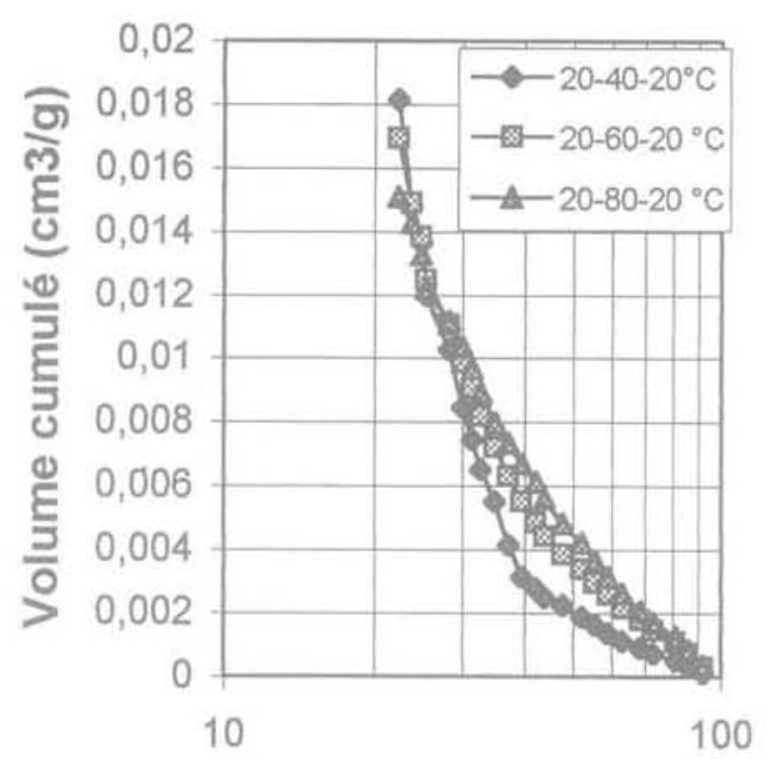

Diamètre des pores (Angströms)

FG. 26 Désorption d'azote sur des échantillons de smectite consolidée sur un chemin cedométrique à $15 \mathrm{MPa}$ après un cycle thermique.

Macro-porosity of smectite samples consolidated at $15 \mathrm{MPa}$ and after thermal cycle. 


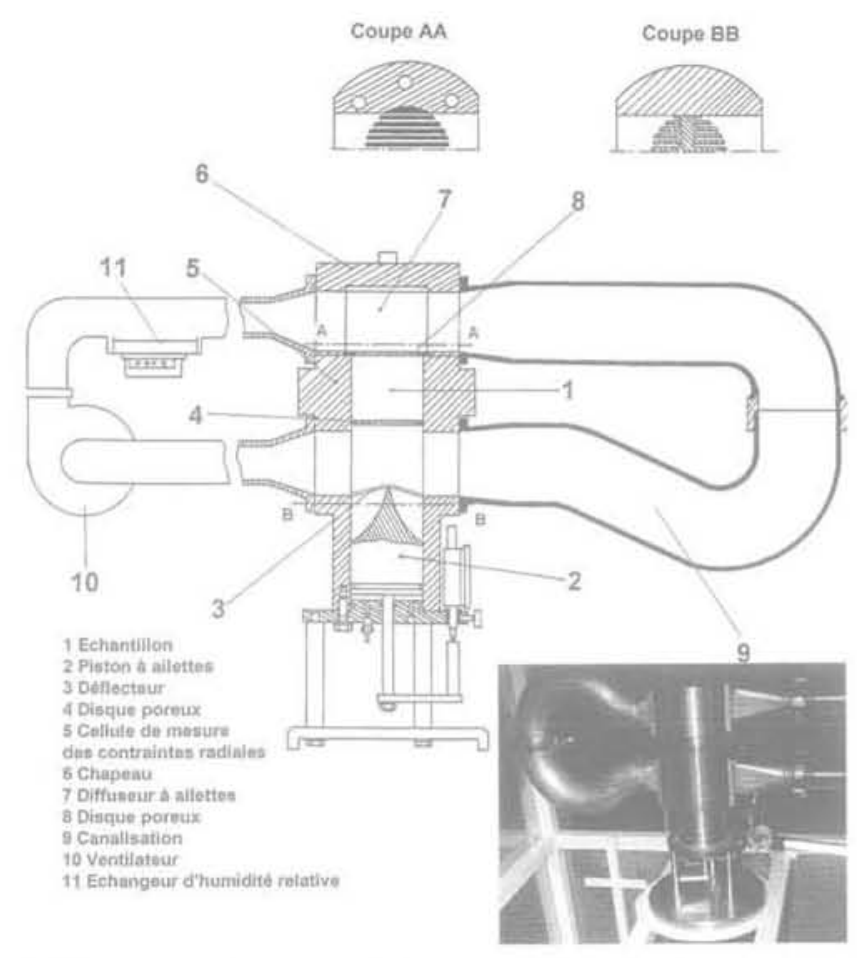

FG. 27 Coupe schématique de la cellule œdométrique à haute pression à succion contrôlée.

Schematic section of high pressure oedometric cell with suction control.

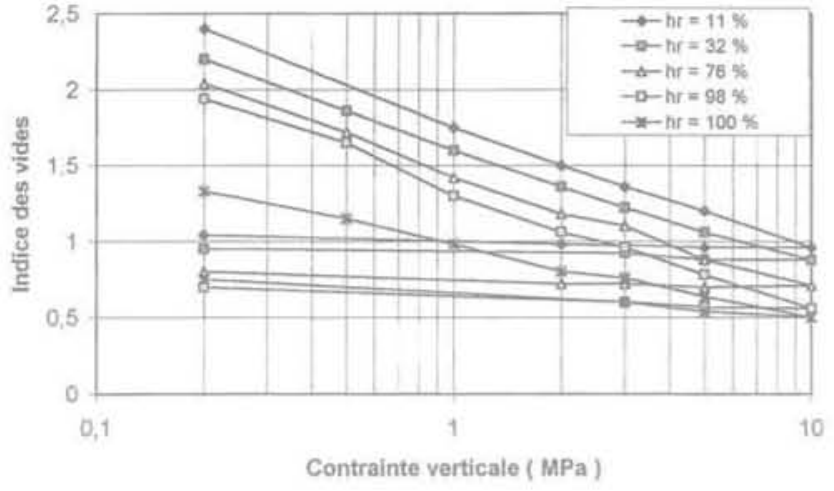

FG. 28 Essais œdométriques à différentes humidités relatives sur une poudre de kaolinite.

Oedometric tests on kaolinite powder at different relative moistures.

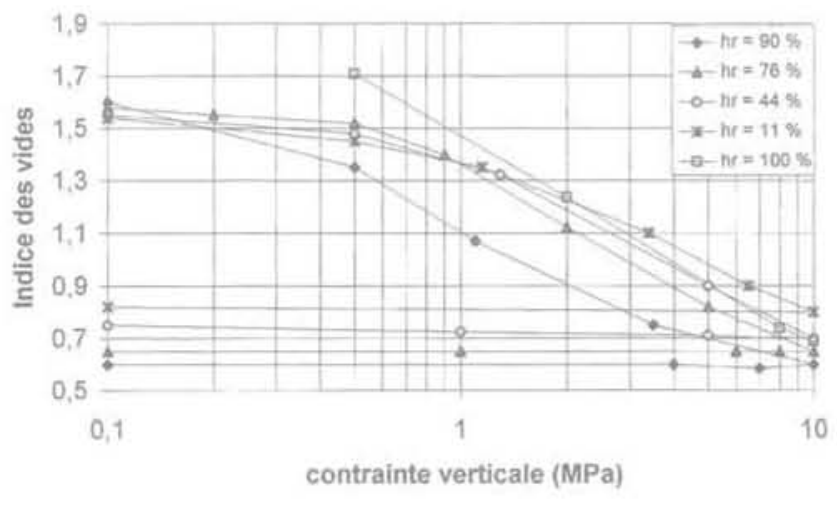

FG. 29 Essais œdométriques à différentes humidités relatives sur une poudre de smectite.

Oedometric tests on smectite powder at diffirent relative moistures.

de décharge n'existent donc que pour des états hydriques proches de la saturation.

\section{Ary.2.}

\section{Cas des essais désorption-sorption à contrainte verticale constante}

Les essais sont réalisés à partir d'une boue de smectite, de kaolinite ou d'argile de Boom. Après consolidation des matériaux jusqu'à $5,5 \mathrm{MPa}$ pour l'argile de Boom, $5 \mathrm{MPa}$ pour la smectite et 2,5 MPa pour la kaolinite, le cycle hydrique se déroule sous contrainte verticale constante. Les figures $30,31,32,33,34$ et 35 traduisent l'évolution des humidités relatives dans la cellule et dans le circuit de ventilation ainsi que celle de l'indice des vides avec la succion. Nous observons dans les trois cas des phénomènes similaires :

- dans la première partie de la désorption, pour des humidités relatives imposées de $100 \%, 98 \%$ et $85 \%$, l'humidité relative mesurée dans la cellule reste constante et proche de la saturation, alors que le matériau subit une forte consolidation hydrique ;

- dans la seconde partie de la désorption, pour des humidités relatives imposées de $66 \%, 33 \%$ ou $11 \%$, l'humidité relative dans la cellule chute pour atteindre une asymptote correspondant à une humidité relative

légèrement supérieure à celle imposée, alors que l'indice des vides ne varie presque plus ;

- la sorption réalisée en une seule étape montre une augmentation continue de l'humidité relative dans la cellule, associée à un léger gonflement du matériau. proportionnel au pouvoir de gonflement de l'argile.

Le premier stade de la désorption correspond à un domaine proche de la saturation où la phase air n'est pas continue, et ne permet donc pas la transmission de la pression de vapeur d'eau jusqu'au capteur installé au centre de la galette. Dans ce domaine l'augmentation des contraintes effectives est due à l'augmentation des potentiels d'eau négatifs et engendre une consolidation du matériau. Le second stade correspond à un domaine très désaturé, possédant une phase air continue et une phase eau condensée et discontinue. Dans ce domaine le concept des contraintes effectives n'est plus applicable, les déformations qui y sont générées sont quasiment réversibles. La frontière entre les deux domaines est caractérisée par une pression d'entrée air dans le matériau qui correspond à la pression interstitielle de désaturation. 

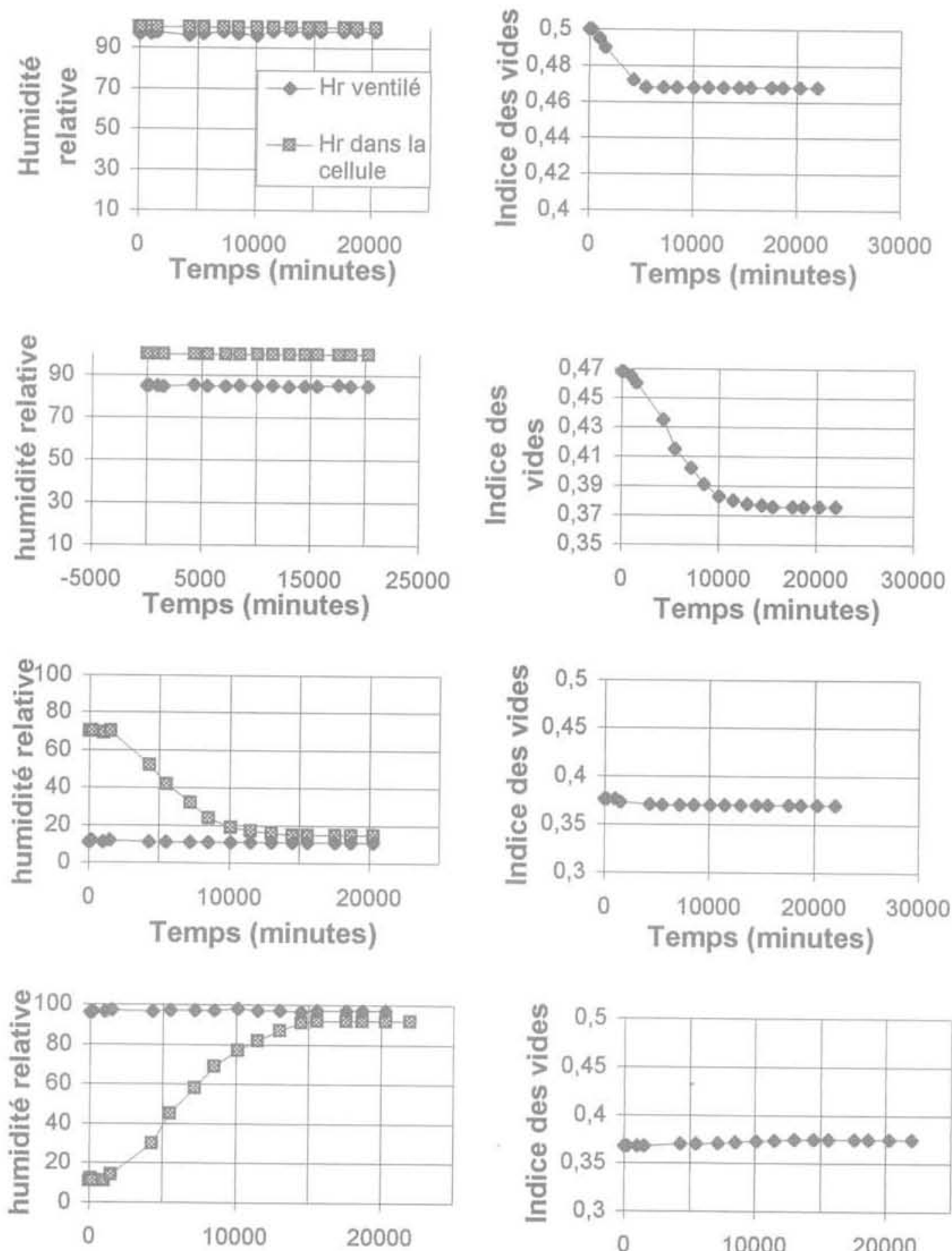

Temps (minutes)

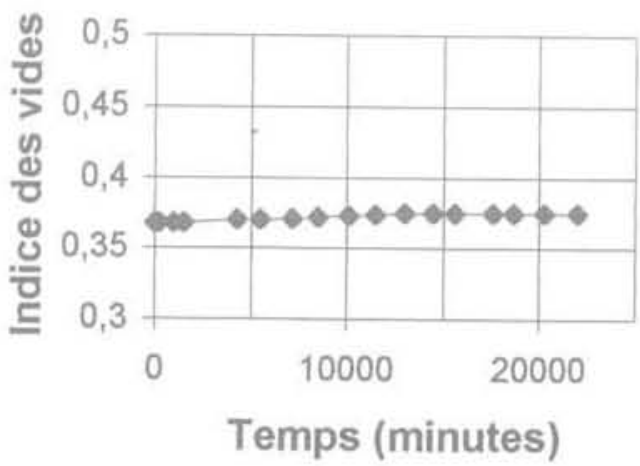

FG. 30 Évolution de l'humidité relative dans l'échantillon et dans l'air ventilé ainsi que de l'indice des vides en fonction du temps pour un échantillon d'argile de Boom. Evolution of relative moisture on the sample and of the ventilled air and evolution of void ratio versus time for a Boom clay sample. 

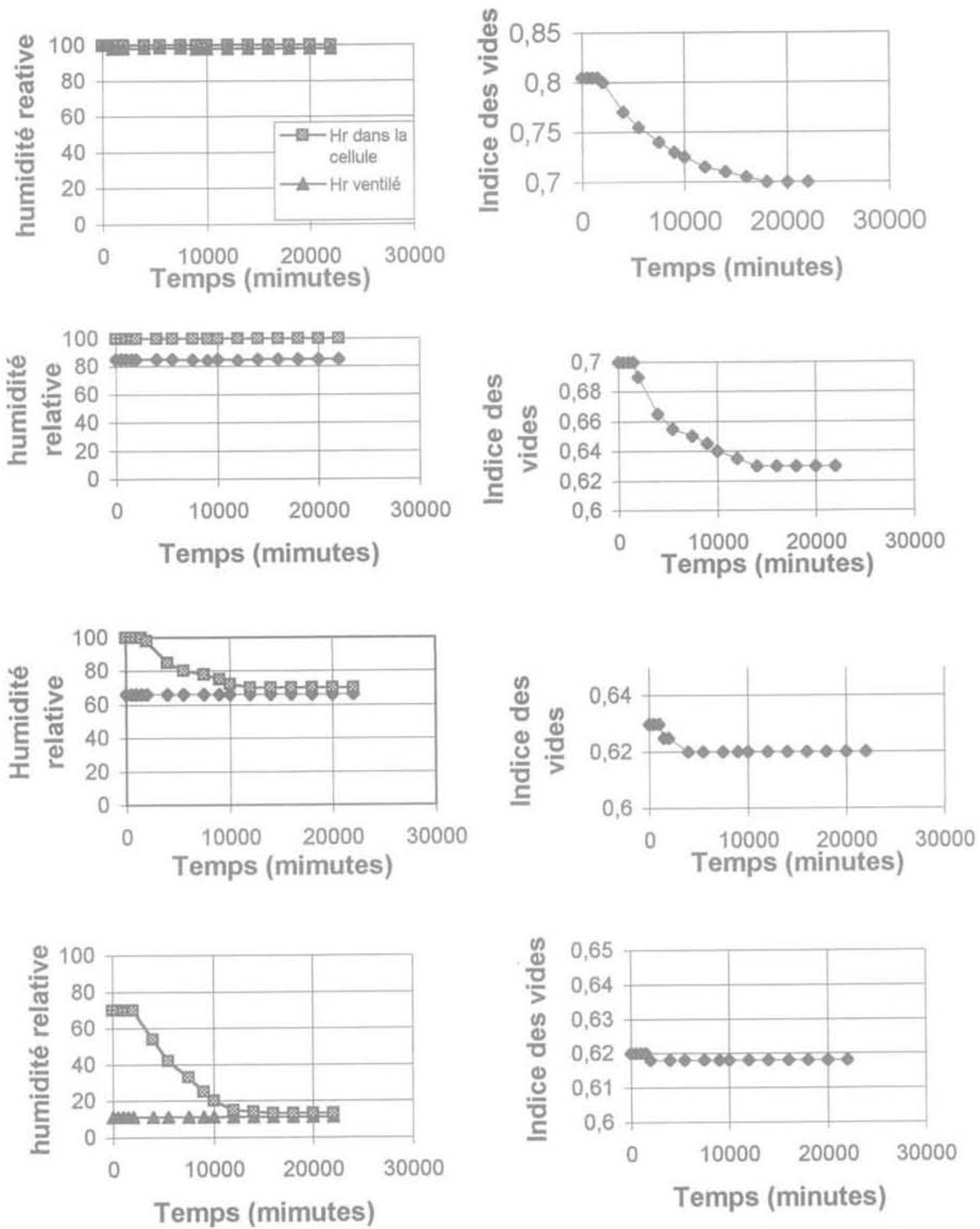

FG.31 Évolution de I'humidité relative dans l'échantillon et dans l'air ventilé ainsi que de Y'indice des vides en fonction du temps pour un échantillon de smectite consolidé à 5 MPa.

Evolution of relative moisture on the sample and of the ventilled air and evolution of void ratic versus time for a smectite sample consolidated at $5 \mathrm{MPa}$. 

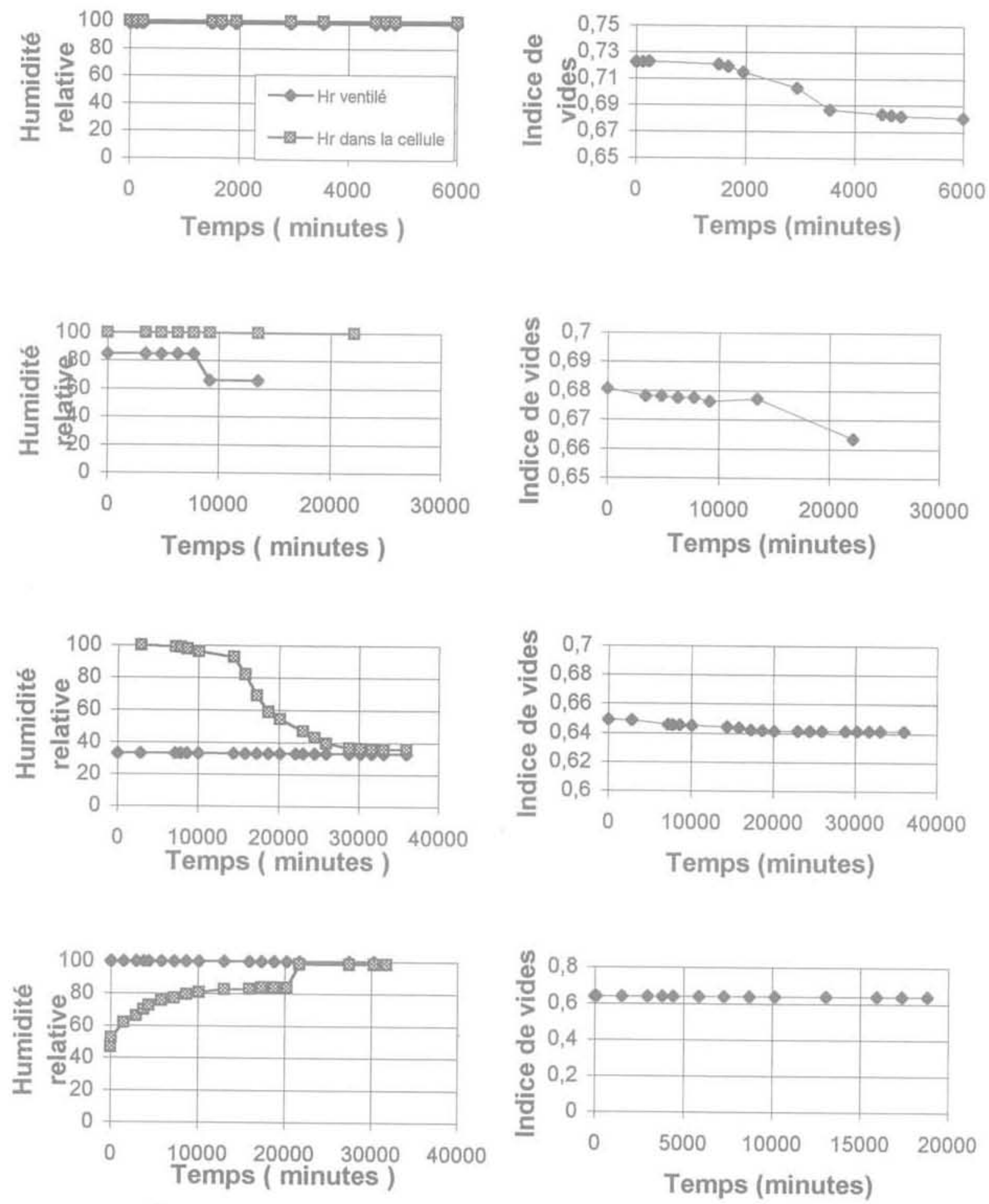

FiG. 32 Evolution de l'humidité relative dans l'échantillon et dans l'air ventilé ainsi que de l'indice des vides en fonction du temps pour un échantillon de kaolinite consolidée à 2,5 MPa. Evolution of relative moisture on the sample and of the ventilled air and evolution of void ratio versus time for a kaollnite sample consolidated at $2.5 \mathrm{MPa}$. 


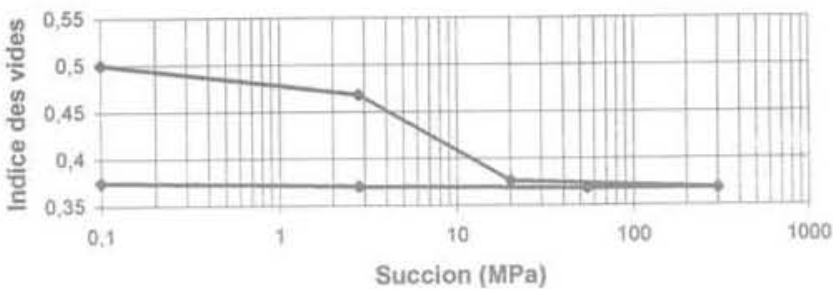

FIG. 33 Variation de l'indice des vides en fonction de la succion pour un échantillon d'argile de Boom sous contrainte verticale constante de $5.5 \mathrm{MPa}$.

Void ratio variations with the suction for a Boom clay sample under a constant vertical loading of $5.5 \mathrm{MPa}$.

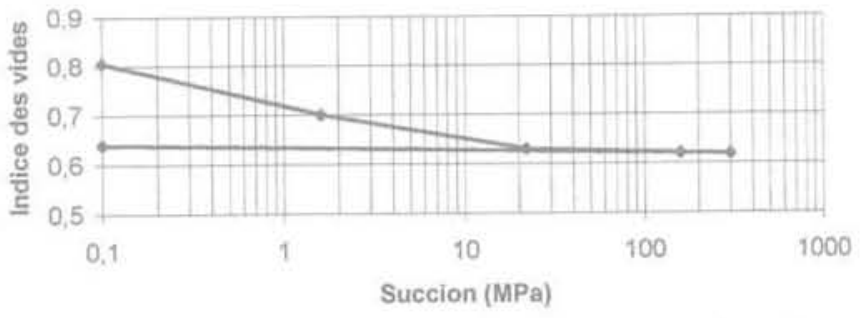

Fig. 34 Variation de l'indice des vides en fonction de la succion pour un échantillon de smectite sous contrainte verticale constante de $5 \mathrm{MPa}$.

Void ratio variations with the suction for $\mathrm{a}$ smectite sample under a constant vertical loading of $5 \mathrm{MPa}$.

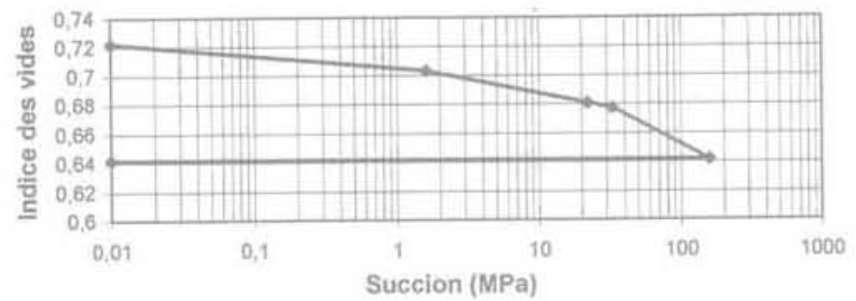

FIG. 35. Variation de l'indice des vides en fonction de la succion pour un échantillon de kaolinite sous contrainte verticale constante de $2,5 \mathrm{MPa}$.

Void ratio variations with the suction for a kaolinite sample under a constant vertical loading of $2.5 \mathrm{MPa}$

\section{2}

\section{Comportement hydro-mécanique sur chemin triaxial drainé}

Des essais triaxiaux drainés à différentes succions ont été réalisés sur des échantillons fabriqués à partir d'une poudre de smectite stabilisée dans des dessicateurs à humidité relative constante.

Les résultats expérimentaux ont été tracés dans les plans $\left(q-\varepsilon_{1}\right)\left(\varepsilon_{3}-\varepsilon_{1}\right)$ et (q-P') (Fig. 36). L'analyse de ces résultats fait apparaître que:
- l'augmentation de la succion entraîne un accroissement de la contrainte déviatoire à la rupture ;

- les pentes de rupture sont quasiment parallèles, l'angle de frottement et la pente d'état critique sont donc indépendants de l'état hydrique du matériau. La cohésion croît par contre avec la succion ;

- pour les états de contraintes très surconsolidés, le comportement du matériau est de type contractant comme pour les argiles gonflantes saturées.

\section{3}

\section{Étude de l'anisotropie des déformations sous sollicitations hydriques}

Un échantillon de smectite, fabriqué par compactage cdométrique à une pression de $15 \mathrm{MPa}$ à partir d'une boue, a subi une désorption en étant placé dans des dessiccateurs à différentes humidités relatives allant de 100 à $33 \%$. Les déformations radiales et axiales sont mesurées à la fin de chaque étape. Les résultats obtenus, présentés sur la figure 37 , montrent que les courbes de variation des déformations axiales et radiales de l'échantillon ont la même forme. Cependant, les déformations axiales sont plus importantes que les déformations radiales. Les déformations introduites lors d'une désorption sont donc anisotropes.

\section{5}

\section{Conclusion et discussion des résultats}

L'étude de la compressibilité œedométrique menée sur quatre types d'argiles saturées et remaniées montre que :

- quel que soit le type d'argile, gonflante ou non, le comportement en charge est élasto-plastique;

- en décharge, le comportement est élastique non linéaire pour les argiles non gonflantes (argile du Bassin parisien et kaolinite) et élasto-plastique pour les argiles gonflantes (Boom et smectite) ;

- la pente de décharge augmente avec la pression de consolidation et la boucle d'hystérésis est d'autant plus large que l'argile est gonfiante.

L'analyse des résultats des essais triaxiaux, non drainés, réalisés sur la kaolinite et la smectite remaniées et saturées à différentes pressions de confinement semble montrer que les fortes pressions de consolidation ne remettent pas en cause le concept d'état critique.

Les résultats expérimentaux des déformations des textures sous les effets mécaniques ont mis en évidence que :

- sous l'effet d'un champ de contraintes mécaniques, les déformations macroscopiques proviennent du compactage des mésopores et des macropores;

- les micropores ne sont affectés ni en taille, ni en volume par les contraintes mécaniques.

On déduit de ces résultats que la première phase de consolidation d'une boue argileuse affecte tous les espaces poreux : interfoliaire, interparticulaire et interagrégat. Au-delà de ces valeurs, la consolidation s'effectue par diminution en taille et en volume des 

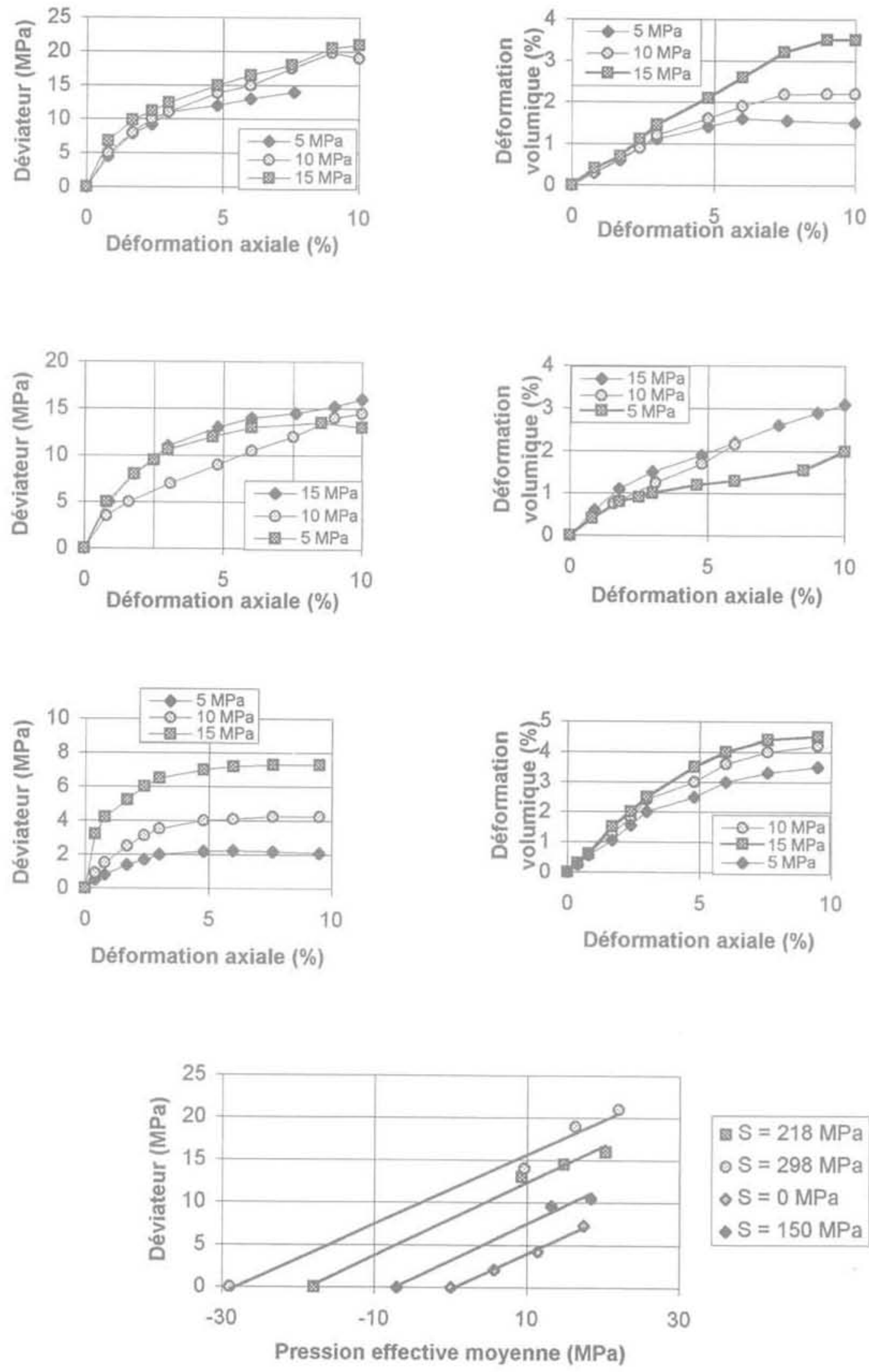

Fis. 36 Essais triaxiaux à différentes succions sur la smectite. Triaxial tests at different suctions on smectite. 


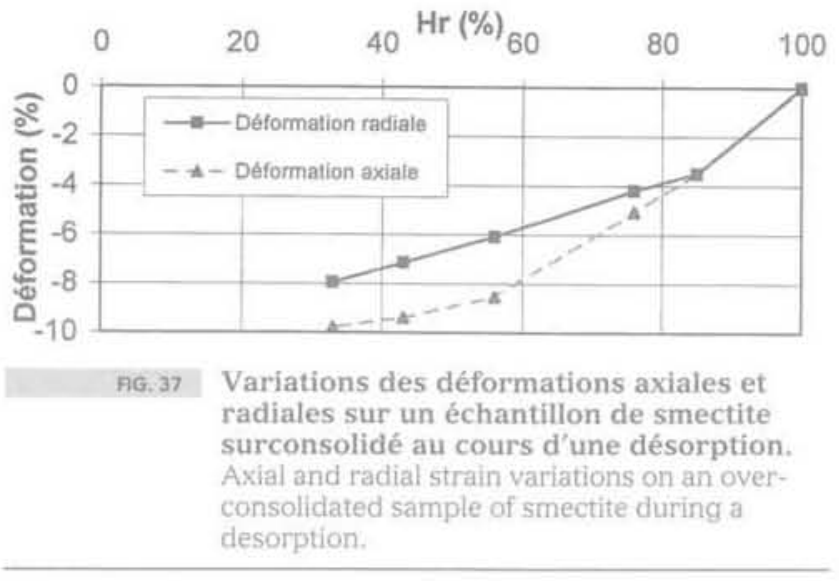

macroporosités interagrégats. Ainsi, la pression de répulsion générée dans les espaces interfoliaires et interparticulaires (des micropores) reste constante. Les variations dies contraintes extérieures produisent alors une variation des contraintes de contact $\left(\delta \sigma_{\text {exterimes }}=\right.$ $\left.\delta \sigma_{\text {contact }}\right)$.

Les contraintes internes se manifestent en décharge. La décharge mécanique provoque, pour les argiles gonflantes, un déséquilibre transitoire entre les contraintes internes de répulsion et les contraintes de contact. L'excès des contraintes de répulsion est ensuite équilibré par la déformation des microporosités. La dilatance des espaces poraux et interfoliaires et interparticulaires induit un gonflement des agrégats et une réorganisation des espaces interagrégats. En définitive, les déformations des microporosités (de nature réversible) jouent en quelque sorte un rôle de catalyse vis-à-vis des déformations des macroporosités (irréversibles). Dans une telle configuration, la variation des contraintes extérieures est compensée par la variation des contraintes de répulsion et d'attraction $\left(\delta \sigma_{\text {entérieure }}=\right.$ $\delta \sigma \mathrm{R}-\mathrm{A})$.

Il en résulte que les déformations irréversibles d'une argile gonflante proviennent des déformations de macroporosités :

i) classiquement dans la phase de consolidation: gats.

ii) en décharge par le gonflement interne des agré-

L'étude expérimentale du comportement thermomécanique que nous avons menée sur quatre argiles saturées et remaniées, nous a permis de mettre en évidence certains des principaux phénomènes liés à l'influence de la température sur le comportement mécanique des argiles. Nous pouvons les résumer dans ce qui suit :

- le chauffage d'un matériau argileux préconsolidé provoque une diminution de son domaine élastique :
- la résistance mécanique des argiles diminue avec laugmentation de la température ;

- à l'état normalement consolidé, un cycle thermique induit un écrouissage positif du matériau. Celuici est d'autant plus important que l'argile est de nature gonflante (smectite) ;

- à l'état surconsolidé, un cycle thermique induit un écrouissage négatif du matériau. Son ampleur est d'autant plus importante que l'argile est gonflante.

Dans le cas des sollicitations thermiques les résultats des mesures de porosimétrie au mercure et de BET indiquent que :

- les micropores ne sont pas affectés par la température ;

- la distribution et le pourcentage des mésopores ne sont pas modifiés.

Par ailleurs, l'analyse des essais cycliques thermiques sur une smectite sous contrainte extérieure constante montre que les déformations d'origine thermique sont anisotropes. Il en résulte que les contraintes thermiques correspondant aux déformations sont nécessairement tensorielles. Elles peuvent être décrites par une contrainte thermique tensorielle $\sigma_{\mathrm{T}}$

L'étude hydro-mécanique réalisée sur trois argiles à l'état partiellement saturé a mis en évidence que :

- l'augmentation de la succion sur les matériaux consolidés produit une augmentation de la pression de consolidation :

- pour des succions inférieures à la pression d'entrée d'air (phase eau continue), la désorption sur des matériaux à l'état normalement consolidé produit une consolidation élasto-plastique ;

- pour des succions supérieures à la pression d'entrée d'air (phase eau discontinue), la désorption des matériaux normalement consolidés provoque des déformations réversibles :

- la résistance mécanique des argiles augmente avec la désaturation :

- à la rupture, il apparaît une cohésion capillaire dont l'amplitude dépend de la succion.

Les essais de désorption sur des galettes de smectite surconsolidée ont montré que les déformations macroscopiques sont anisotropes. Cette anisotropie peut être traduite par une contrainte tensorielle capillaire $\sigma_{\text {cap }}$.

Ainsi sur la base de ces constatations, nous proposons une extension des contraintes effectives aux sollicitations thermo-hydro-mécaniques, Les contraintes extérieures sont équilibrées par les contraintes de contact pour les états normalement consolidés ou de répulsion pour les états surconsolidés, les contraintes capillaires et les contraintes thermiques:

$$
\underline{\sigma_{\text {ext }}}=\underline{\sigma_{c}}+\underline{\sigma_{\text {cap }}}+\underline{\sigma_{I}}(\mathrm{OCR}=1)
$$

et $\underline{\sigma_{\text {ext }}}=\underline{\sigma_{\mathrm{R}}}+\underline{\sigma_{\text {cap }}}+\underline{\sigma_{\mathrm{T}}}(\mathrm{OCR}>1)$. 
Aldrich - Catalog handbook of fine chemicals. Aldrich Chemical Compagny Inc., Milwaukee, 1986-1987.

Baldi G., Borsetto M., Hueckel T. - Calibration of mathematical models for the simulation of thermal, seepage and mechanical behaviour of Boom clay. CEC publishers, Luxembourg EUR 10924 EN, p. 215, 1987

Baldi G., Hueckel T. Peano A., Pellegrini R. - Development in modelling of the thermo-hydro-geomechanical behaviour of Boom clay and clay based buffer materials. Final report for CEC ISMES, Bergamo, Italy, 1990.

Ballivy G. - Contribution à l'êtude du rổle de l'eau dans le comportement mècanique des roches fissurées. Thèse de doctorat ès sciences appliquées à l'École polytechnique de Montréal, 266 p.r 1976.

Ballivy G., Daoud M. Saleh K. - Comportement physico-mécanique des roches sous l'effet de l'humidité relative ambiante. Structure et comporternent mécanique des géomatériaux. Colloque René-Houpert, p. 247-256, 1992.

Börgesson L., Karnland O., Johansnesson L.E. - Modelling of the physical behaviour of clay barriers close to water saturation. International workshop on Thermo-mechanics of clav and clay barriers, Bergamo, Italy, 1993.

Colback P.S.B., Wiid B.L. - The influence of moisture content on the compressive strength of rocks. Third Canadian Symposium on Rock Mechanics, Toronto. p. $65-83,1965$

Daian J.F. - Processus de condensation et de transfert d'eau dans un matériau méso- et macroporeux. Étude expérimental du mortier de ciment. Thèse de doctorat d'Etat, Institut national polytechnique ef université de Grenoble. 1986.

Def Olmo C., Fioravante V., Gera F., Hueckel T.. Mayor J.C. Pellecrini R. - Thermomechanical properties of cleep argillaceous formations. Engineering Geology, 41, p. 87-101, 1996.

Felix B., Lebon P., Miguez, Plas F. - A review of Andra's research pro. grammes on the thermo-hydromechanical behaviour of clay in connection with the radioactive waste disposal project in deep geological formations. Engineering Geology, 41, p. 35-50, 1996.

Lingnau B.E., Yarechewski D., Graharn J. Tanaka N.. Gray M.N. - Stress-strain properties of sand bentonite buffer at elevated temperatures. International workhop on Thermo-mechanics of clay and clay barriers, Bergamo, Italy, 1993

Piau J.-M. - Compaction and subsidence of petroleum reservoirs. Mechanics of porous media, Charlez (ed.), Balkema. p. 215-244, 1995.

Robinet J.-C. Rahbaoul A.. Plas F. Lebon $\mathrm{P}$. - A constitutive thermomecha nical model for saturated clays. Engineering Geology, 41, D. 145-169, 1996

Robinet J.-C., Pakzad M., Plas F. - Un modèle rhéologique pour les argiles gonflantes. Revue Francaise de Géotechnique, $\mathrm{n}^{\circ} 62$, p. 57-67, 1994.

Robinet J.-C., Pasquiou A., Palut J.-M. Noynert N. - Phenomenology of hydirical exchanges between underground atmosphere and storage host-rock. Final Report (E.C) (ed.), 1997.

Robinet J.-C. Lecomte P. - Dispositif cedométrique haute pression à température et hygromẻtrie contrỏlées. Brevet d'invention, $n^{\circ} 97$ 03564, 1997.

Rousset G. - Comportement mécanique des argiles profondes, application au stockage de déchets radioactifs. Thèse de doctorat ENPC. Paris, 1998. 